\title{
Differential Operator Lie Algebras on the Ring of Laurent Polynomials
}

\section{Liang Chen}

Department of Mathematics, University of Alberta, Edmonton (Alberta) T6G 2G1, Canada

Received: 15 September 1993/in revised form: 6 April 1994

Abstract: A class of differential operator Lie algebras on the unit circle is introduced and discussed. They are the natural generalizations of the Witt algebra and the Virasoro algebra. Among them are the higher-spin algebras $W_{1+\infty}$ and $W_{\infty}$ which occur in the physics literature.

\section{Introduction}

The Witt algebra $W$ is the complex Lie algebra of polynomial vector fields on the unit circle $S^{1}$. An element of $W$ is a linear combination of the elements of the form $e^{i n \theta} \frac{d}{d \theta}$, where $\theta$ is a real parameter, and the Lie bracket of $W$ is given by

$$
\left[e^{i m \theta} \frac{d}{d \theta}, e^{i n \theta} \frac{d}{d \theta}\right]=i(n-m) e^{i(m+n) \theta} \frac{d}{d \theta} .
$$

If we define $t=e^{i \theta}$, then the elements $t^{m}=e^{i m \theta}, m \in \mathbb{Z}$, span the Laurent polynomial ring $\mathbb{C}\left[t, t^{-1}\right]$, and $e^{i m \theta} \frac{d}{d \theta}=i t^{m+1} \frac{d}{d t}$ may be viewed as a first order differential operator on $\mathbb{C}\left[t, t^{-1}\right]$. Let $d_{m}=t^{m+1} \frac{d}{d t}$. Then $W=\sum_{m \in \mathbb{Z}} \mathbb{C} d_{m}$, and $\left[d_{m}, d_{n}\right]=(n-m) d_{m+n}$. The Virasoro algebra is $V i r=\sum_{m \in \mathbb{Z}} \mathbb{C} L_{m}+\mathbb{C} \phi$ with

$$
\begin{aligned}
{\left[L_{m}, L_{n}\right] } & =(n-m) L_{m+n}+\frac{m^{3}-m}{12} \delta_{m+n, 0} \phi, \\
{\left[L_{m}, \phi\right] } & =0 .
\end{aligned}
$$

Recently, C.N. Pope and X. Shen [PS], C.N. Pope, L.J. Roman and X. Shen [PRS1], [PRS2] studied the higher spin algebras $W_{\infty}$ and $W_{1+\infty}$, the generalizations of $W$. The Lie algebra $W_{1+\infty}$ has basis $V_{m}^{i}$, where $m \in \mathbb{Z}, i \in \mathbb{Z} \geqq 0$, and Lie bracket

$$
\begin{aligned}
{\left[V_{m}^{i}, V_{n}^{j}\right]=} & g_{0}^{i j}(m, n ; \mu) V_{m+n}^{i+j}+q^{2} g_{2}^{i j}(m, n ; \mu) v_{m+n}^{i+j-2}+\cdots \\
& +q^{2 r} g_{2 r}^{i j}(m, n ; \mu) v_{m+n}^{i+j-2 r}+\cdots+q^{2 i} c_{i}(m ; \mu) \delta^{i j} \delta_{m+n, 0},
\end{aligned}
$$


where $V_{m}^{i}$ corresponds to the $m$ th Fourier mode of a conformal spin $i+2$ field, $q$ is a parameter, $c_{i}(m ; \mu)$ are the central terms. The structure constants are given by

$$
\begin{aligned}
g_{2 r}^{i j}(m, n ; \mu)= & \frac{\phi_{2 r}^{i j}(\mu)}{2(2 r+1) !} N_{2 r}^{i j}(m, n) \\
N_{2 r}^{i j}(m, n)= & \left.\sum_{k=0}^{2 r+1}(-1)^{k} \underset{k}{2 r+1}\right)[i+1+m]_{2 r+1-k} \\
& \times[i+1-m]_{k}[j+1+n]_{k}[j+1-n]_{2 r+1-k} \\
{[a]_{n}=} & \frac{a !}{(a-n) !} .
\end{aligned}
$$

If we parameterize $\mu$ in terms of a variable $s$ by $\mu=s(s+1)=-\frac{1}{4}$, then $\phi_{2 r}^{i j}(\mu)$ can be expressed as

$$
\phi_{2 r}^{i j}(\mu)={ }_{4} F_{3}\left[-\frac{1}{2}-2 s, \frac{3}{2}+2 s,-r-\frac{1}{2},-r,-i-\frac{1}{2},-j-\frac{1}{2}, i+j-2 r+\frac{5}{2} ; 1\right],
$$

where ${ }_{4} F_{3}$ is a generalized hypergeometric function (see [PRS3] for details). We note that $W_{\infty}$ is a Lie subalgebra of $W_{1+\infty}$.

Later, [PRS4] proved that $W_{1+\infty}$ is nothing but the algebra of all polynomial differential operators on the unit circle, including differential operators of arbitrary order, namely

$$
\mathbb{C}\left[t, t^{-1}, \frac{d}{d t}\right]=\sum_{m \in \mathbb{Z}, r \in \mathbb{Z}_{\geqq 0}} \mathbb{C} t^{m}\left(\frac{d}{d t}\right)^{r} .
$$

This brought a connection between the higher spin algebras and the algebra of all smooth differential operators on the unit circle. For earlier discussion, see also [Sa].

Note that the Lie bracket of $W_{1+\infty} \cong \mathbb{C}\left[t, t^{-1}, \frac{d}{d t}\right]$ given above is very complex. We rechoose a basis for $\mathbb{C}\left[t, t^{-1}, \frac{d}{d t}\right]$ as follows: For all $m \in \mathbb{Z}, r \in \mathbb{Z} \geqq 0$, define

$$
d_{m}^{r}:=t^{m}\left(t \frac{d}{d t}\right)^{r}
$$

Then $\left\{d_{m}^{r} \mid m \in \mathbb{Z}, r \in \mathbb{Z} \geqq 0\right\}$ is a basis of $\mathbb{C}\left[t, t^{-1}, \frac{d}{d t}\right]$. The Lie bracket is

$$
\left[d_{m}^{r}, d_{n}^{s}\right]=\sum_{k=0}^{r+s}\left(\left(\begin{array}{l}
r \\
k
\end{array}\right) n^{k}-\left(\begin{array}{l}
s \\
k
\end{array}\right) m^{k}\right) d_{m+n}^{r+s-k} .
$$

From this we see that $\mathbb{C}\left[t, t^{-1}, \frac{d}{d t}\right]$ has $\mathbb{Z}$-grading

$$
\mathbb{C}\left[t, t^{-1}, \frac{d}{d t}\right]=\sum_{m \in \mathbb{Z}} \mathfrak{g}_{m},\left[\mathfrak{g}_{m}, \mathfrak{g}_{n}\right] \subseteq \mathfrak{g}_{m+n},
$$

where the graded subspace $\mathfrak{g}_{m}:=\sum_{r=0}^{\infty} \mathbb{C} d_{m}^{r}$. Further, the $\mathbb{C}$-linear map $\sigma$ such that

$$
\sigma\left(d_{m}^{r}\right)=d_{m+1}^{r} \forall m \in \mathbb{Z}, r \in \mathbb{Z}_{\geqq 0}
$$

is bijective from $\mathbb{C}\left[t, t^{-1}, \frac{d}{d t}\right]$ to itself and shifts a graded subspace to another. We call $\sigma$ a shift operator of $\mathbb{C}\left[t, t^{-1}, \frac{d}{d t}\right]$ (see Sect. 1 for more details). A Lie subalgebra 
$\mathfrak{h}$ of $\mathbb{C}\left[t, t^{-1}, \frac{d}{d t}\right]$ is called a homogeneous Lie subalgebra if

$$
\mathfrak{h}=\sum_{m \in \mathbb{Z}} \mathfrak{h}_{m},
$$

where $\mathfrak{h}_{m}=\mathfrak{h} \cap \mathfrak{g}_{m}$, and $\sigma(\mathfrak{h})=\mathfrak{h}$.

In this paper we investigate the homogeneous Lie subalgebras of $\mathbb{C}\left[t, t^{-1}, \frac{d}{d t}\right]$ and their representations. In Sect. 1, we give the classification of the homogeneous Lie subalgebras and find that, except for a few examples, these Lie subalgebras are determined by polynomials in some way. The information from the automorphism group of $\mathbb{C}\left[t, t^{-1}, \frac{d}{d t}\right]$ is important for the study of the structure of $\mathbb{C}\left[t, t^{-1}, \frac{d}{d t}\right]$ and its Lie subalgebra. Since homogeneous Lie subalgebras are $\mathbb{Z}$-graded, in Sect. 2, we determine the automorphisms of $\mathbb{C}\left[t, t^{-1}, \frac{d}{d t}\right]$ which preserve the $\mathbb{Z}$-grading. Our result is that these automorphisms constitute a group isomorphic to $\left(\mathbb{Z}_{2} \triangleright<(\mathbb{C},+)\right) \times\left(\mathbb{C}^{*}, \cdot\right)$. In Sect. 3, we discuss the algebraic properties of the homogeneous Lie subalgebras. We see that there is a one to one correspondence between the ideal lattice of the ring of one variable polynomials and the homogeneous Lie subalgebras of $\mathbb{C}\left[t, t^{-1}, \frac{d}{d t}\right]$ and almost all of the homogeneous Lie subalgebras are indecomposible. Since the (universal) central extension of $W$, the Virasoro algebra $V i r$, is so important in Mathematics and Physics and $\mathbb{C}\left[t, t^{-1}, \frac{d}{d t}\right]$ is a natural generalization of $W$, it is natural to consider the central extensions of the homogeneous Lie subalgebras. V. Kac and A. Raina [KR] defined the infinite dimensional Lie algebra $\overline{\mathfrak{a}_{\infty}}$ and its 1 -dimensional central extension $\mathfrak{a}_{\infty}$. Then imbedded $W$ as a Lie subalgebra of $\overline{\mathfrak{a}_{\infty}}$ and obtained $V$ ir as a Lie subalgebra of $\mathfrak{a}_{\infty}$. Following Kac and Raina's ideal, in Sect. 4, we imbed $\mathbb{C}\left[t, t^{-1}, \frac{d}{d t}\right]$ as a Lie subalgebra of $\overline{\mathfrak{a}_{\infty}}$ and obtain the 1-dimensional central extensions of the homogeneous Lie subalgebras. Moreover, we discuss the universal central extension of $\mathfrak{g}\{0,1\}:=\sum_{m \in \mathbb{Z}} \mathbb{C} d_{m}^{1}+\sum_{m \in \mathbb{Z}} \mathbb{C} d_{m}^{0}$. On the representation side, for the Virasoro algebra, Kac has the following conjecture: Every irreducible representation of the Virasoro algebra with finite dimensional weight spaces is either highest or lowest weight or has all its weight spaces of dimension less than or equal to one. Kaplansky and Santharoubane [Kap, KS] verified the conjecture in the case when all eigenspaces of $d_{0}$ have dimension $\leqq 1$, and Chari and Pressley [CP] proved the conjecture in the case the representation is unitary. Finally, the conjecture has been proved by Martin and Piard [MP], and Mathieu [Ma]. As a generalization, we define the admissible modules (the modules with finite dimensional weight spaces) of the homogeneous Lie subalgebras and classify the admissible $\mathbb{C}\left[t, t^{-1}, \frac{d}{d t}\right]$-modules with 1-dimensional weight spaces in Sect. 5, and discuss the highest weight modules in Sect. 6.

We denote the complex number field by $\mathbb{C}$, the real number field by $\mathbb{R}$, and the integer ring by $\mathbb{Z}$. All Lie algebras considered are complex Lie Algebras.

\section{Differential Operator Lie Algebras on $\mathbb{C}\left[t, t^{-1}\right]$}

In this section, we consider the algebra of differential operators on the Laurent polynomial ring $\mathbb{C}\left[t, t^{-1}\right]$, namely $\mathbb{C}\left[t, t^{-1}, \frac{d}{d t}\right]$, and give it a Lie algebra structure. We have seen in the Introduction that by suitable choice of basis, we obtain a $\mathbb{Z}$-grading on $\mathbb{C}\left[t, t^{-1}, \frac{d}{d t}\right]$, and hence we have the shift map $\sigma$ on it. We call a Lie subalgebra of $\mathbb{C}\left[t, t^{-1}, \frac{d}{d t}\right]$ which is $\mathbb{Z}$-graded and invariant under the shift map 
a homogeneous Lie subalgebra. The main result in this section is the classification of the homogeneous Lie subalgebras of $\mathbb{C}\left[t, t^{-1}, \frac{d}{d t}\right]$.

As a vector space over $\mathbb{C}, \mathbb{C}\left[t, t^{-1}, \frac{d}{d t}\right]$ has a basis $\left\{d_{m}^{r} \mid m \in \mathbb{Z}, r \in \mathbb{Z} \geqq 0\right\}$, where $d_{m}^{r}:=t^{m}\left(t \frac{d}{d t}\right)^{r}$, and the action of $d_{m}^{r}$ on $\mathbb{C}\left[t, t^{-1}\right]$ is given by

$$
d_{m}^{r} \cdot t^{k}=k^{r} t^{m+k}
$$

for all $k \in \mathbb{Z}$. Let $p(x)=\sum_{i} a_{i} x^{i} \in \mathbb{C}[x]$ be a polynomial and define

$$
d_{m}(p(x))=\sum_{i} a_{i} d_{m}^{i}
$$

Then

Moreover,

$$
\begin{aligned}
d_{m}(p(x)) \cdot t^{k} & =\sum_{i} a_{i} d_{m}^{i} \cdot t^{k} \\
& =\sum_{i} a_{i} k^{i} t^{m+k} \\
& =p(k) t^{m+k}
\end{aligned}
$$

$$
\begin{aligned}
& \left(d_{m}(p(x)) d_{n}(q(x))-d_{n}(q(x)) d_{m}(p(x))\right) \cdot t^{k} \\
& =q(k) d_{m}(p(x)) t^{n+k}-p(k) d_{n}(q(x)) t^{m+k} \\
& =(p(n+k) q(k)-p(k) q(k+m)) t^{m+n+k} \\
& =d_{m+n}(p(x+n) q(x)-p(x) q(x+m)) t^{k}
\end{aligned}
$$

for all $k \in \mathbb{Z}$.

So, if we define

$$
\left[d_{m}(p(x)), d_{n}(q(x))\right]=d_{m+n}(p(x+n) q(x)-p(x) q(x+m)),
$$

then $\mathbb{C}\left[t, t^{-1}, \frac{d}{d t}\right]$ is a Lie algebra. Note that if $p(x)=x^{r}, q(x)=x^{s}$, then the Lie bracket we obtained here is exactly that we have seen in the introduction. We note for future reference that $d_{m}=d_{m}^{1}=d_{m}(x)$ and $d_{m}^{s}=d_{m}\left(x^{s}\right)$. We denote $\mathbb{C}\left[t, t^{-1}, \frac{d}{d t}\right]$ by $g$ in the rest of this paper.

Definition. A Lie subalgebra of $\mathfrak{g}$ is called a differential operator Lie algebra on $\mathbb{C}\left[t, t^{-1}\right]$.

Setting

$$
\mathfrak{g}_{m}=\sum_{r \geqq 0} \mathbb{C} d_{m}^{r},
$$

where $m \in \mathbb{Z}$, then $\mathfrak{g}=\sum_{m \in \mathbb{Z}} \mathfrak{g}_{m}$, and $\left[\mathfrak{g}_{m}, \mathfrak{g}_{n}\right] \subseteq \mathfrak{g}_{m+n}$. So $\mathfrak{g}$ is a $\mathbb{Z}$-graded Lie algebra. In particular, $\left[\mathrm{g}_{0}, \mathfrak{g}_{0}\right]=0$, and $\left[d_{0}^{1}, d_{n}^{s}\right]=n d_{n}^{s}$. We see that $\mathrm{g}_{0}$ is an abelian Lie subalgebra of $\mathfrak{g}$ and $\mathfrak{g}_{m}$ is the eigenspace of $\operatorname{ad}\left(d_{0}^{1}\right)$, the adjoint map, of eigenvalue $m$.

The linear map $\sigma$ from $g$ to itself such that $\sigma\left(d_{m}^{r}\right)=d_{m+1}^{r}$ for all $m \in \mathbb{Z}, r \in \mathbb{Z} \geqq 0$ is called the (canonical) shift of $\mathrm{g}$. Clearly $\sigma$ is one to one and onto. 
Definition. Let $\mathfrak{h} \subseteq \mathfrak{g}$ be a Lie subalgebra. $\mathfrak{h}$ is called a homogeneous Lie subalgebra of $\mathfrak{g}$ if

(i) $\mathfrak{h}=\sum_{m \in \mathbb{Z}} \mathfrak{h}_{m}$, where $\mathfrak{h}_{m}:=\mathfrak{h} \cap \mathfrak{g}_{m}$,

(ii) $\sigma(\mathfrak{h})=\mathfrak{h}$.

The following are examples of homogeneous Lie subalgebras of $\mathfrak{g}$ :

(1) $\mathfrak{g}\{0\}:=\sum_{m \in \mathbb{Z}} \mathbb{C} d_{m}^{0}$ is an abelian Lie subalgebra of $\mathfrak{g}$ :

(2) For any $\alpha \in \mathbb{C}$, define $\mathfrak{g}\{x+\alpha\}:=\sum_{m \in \mathbb{Z}} \mathbb{C} d_{m}(x+\alpha)$. Then

$$
\left[d_{m}(x+\alpha), d_{n}(x+\alpha)\right]=(n-m) d_{m+n}(x+\alpha) .
$$

So $g\{x+\alpha\}$ is isomorphic to the infinite dimensional Witt algebra.

(3) $\mathfrak{g}\{0,1\}:=\sum_{m \in \mathbb{Z}} \mathbb{C} d_{m}^{1}+\sum_{m \in \mathbb{Z}} \mathbb{C} d_{m}^{0}$.

Before we give the classification of the homogeneous Lie subalgebras, we prove the following:

Proposition 1. $\mathfrak{h} \subseteq \mathfrak{g}$ is a homogeneous Lie subalgebra iff there exists a subspace $V \subseteq \mathbb{C}[x]$ satisfying

$$
p(x+n) q(x)-p(x) q(x+m) \in V \quad \forall p(x), q(x) \in V, \quad \forall m, n \in \mathbb{Z},
$$

such that

$$
\mathfrak{h}=\sum_{p(x) \in V, m \in \mathbb{Z}} \mathbb{C} d_{m}(p(x)) .
$$

Proof. Clearly if $V \subseteq \mathbb{C}[x]$ is a subspace satisfying the above condition, then $\sum_{p(x) \in V, m \in \mathbb{Z}} \mathbb{C} d_{m}(p(x))$ is a homogeneous Lie subalgebra of $\mathfrak{g}$. Conversely, assume that $\mathfrak{h} \subseteq \mathfrak{g}$ is a homogeneous Lie subalgebra. Then

$$
\mathfrak{h}=\sum_{m \in \mathbb{Z}} \mathfrak{h}_{m}, \text { where } \mathfrak{h}_{m}=h \cap \mathfrak{g}_{m} .
$$

Note that if $d_{m}(p(x)) \in \mathfrak{h}_{m}$, then $d_{n}(p(x)) \in \mathfrak{h}_{n}$ for all $n \in \mathbb{Z}$ since $\sigma^{k}\left(d_{m}(p(x))\right)=$ $d_{m+k}(p(x))$. Let

$$
V=\left\{p(x) \in \mathbb{C}[x] \mid d_{m}(p(x)) \in \mathfrak{h} \quad \forall m \in \mathbb{Z}\right\} .
$$

Then for polynomials $p(x), q(x) \in V, m, n \in \mathbb{Z}$,

$$
\left[d_{m}(p(x)), d_{n}(q(x))\right]=d_{m+n}(p(x+n) q(x)-p(x) q(x+m)) \in \mathfrak{h} .
$$

Hence

$$
p(x+n) q(x)-p(x) q(x+m) \in V, \quad \forall m, n \in \mathbb{Z} .
$$

$V$ satisfies the required condition.

Remark. By Proposition 1, classifying the homogeneous Lie subalgebras of $g$ is equivalent to classifying the subspaces of $\mathbb{C}[x]$ which satisfy the condition given in the proposition. 
Proposition 2 (Classification of homogeneous Lie subalgebras). The following are all of the homogeneous Lie subalgebras of $\mathfrak{g}$ :

(1) $\mathfrak{g}\{0\}, \mathfrak{g}\{0,1\}, \mathfrak{g}\{x+\alpha\}$ where $\alpha \in \mathbb{C}$.

(2) $\mathfrak{g}_{\langle p(x)\rangle}:=\sum_{q(x) \in\langle p(x)\rangle, m \in \mathbb{Z}} \mathbb{C} d_{m}(q(x))$, where $\langle p(x)\rangle$ is the ideal of $\mathbb{C}[x]$ generated by $p(x)$.

Proof. We have seen that $\mathfrak{g}\{0\}, \mathfrak{g}\{0,1\}, \mathfrak{g}\{x+\alpha\}$ are all homogeneous Lie subalgebras. Now for any $p(x) \in \mathbb{C}[x]$, if $q(x), r(x) \in\langle p(x)\rangle$, then clearly

$$
q(x+n) r(x)-q(x) r(x+m) \in\langle p(x)\rangle,
$$

for all $m, n \in \mathbb{Z}$. So $\mathfrak{g}_{\langle p(x)\rangle}$ is a homogeneous Lie subalgebra of $\mathfrak{g}$ by Proposition 1 . Conversely, let $V \subseteq \mathbb{C}[x]$ be a subspace such that

$$
p(x+n) q(x)-p(x) q(x+m) \in V \quad \forall p(x), q(x) \in V, \quad \forall m, n \in \mathbb{Z} .
$$

Define $\mathfrak{g}_{V}:=\sum_{m \in \mathbb{Z}, p(x) \in V} \mathbb{C} d_{m}(p(x))$.

(a) Suppose for all $p(x) \in V, \operatorname{deg}(p(x))=0$. Then $V=\mathbb{C}$ and $\mathfrak{g}_{V}=\mathfrak{g}\{0\}$.

(b) Suppose for all $p(x) \in V, \operatorname{deg}(p(x)) \leqq 1$, but there exists $p(x) \in V$ with degree 1. Assume that $p(x)=x+\alpha \in V$. If there exists $\beta \in \mathbb{C}$ such that $\beta \neq \alpha$ and $x+\beta \in$ $V$, then $V=\mathbb{C} x+\mathbb{C}$ and $\mathfrak{g}_{V}=\mathfrak{g}\{0,1\}$. Otherwise, $V=\mathbb{C}(x+\alpha)$ and $\mathfrak{g}_{V}=\mathfrak{g}\{x+\alpha\}$.

(c) Suppose there exists $q(x) \in V$ such that $\operatorname{deg}(q(x)) \geqq 2$.

Let

$$
p(x)=x^{r}+a_{r-1} x^{r-1}+\cdots+a_{0} \in V
$$

be the choice with minimal degree and let

$$
q(x)=x^{s}+b_{s-1} x^{s-1}+\cdots+b_{0} \in V,
$$

where $s \geqq 2$.

Case 1: $r=0$. By the Taylor formula,

$$
q(x+m)=q(x)+q^{\prime}(x) m+\frac{q^{(2)}(x)}{2 !} m^{2}+\cdots+\frac{q^{(s)}(x)}{s !} m^{s} .
$$

Since $q(x+m)-q(x)=q^{\prime}(x) m+\frac{q^{(2)}(x)}{2 !} m^{2}+\cdots+\frac{q^{(s)}(x)}{s !} m^{s} \in V$, for all $m \in \mathbb{Z}$, and the determinant of

$$
\left(\begin{array}{cccc}
1 & 1 & \cdots & 1 \\
2 & 2^{2} & \cdots & 2^{s} \\
\vdots & \vdots & \cdots & \vdots \\
s & s^{2} & \cdots & s^{s}
\end{array}\right)
$$

is nonzero, $q^{\prime}(x), \frac{q^{(2)}(x)}{2 !}, \ldots, \frac{q^{(s)}(x)}{s^{\prime}} \in V$. It follows that $1, x, x^{2}, \ldots, x^{s-1} \in V$. But $q(x) \in V$, so also $x^{s} \in V$. Assume that $1, x, \ldots, x^{k} \in V, k \geqq s \geqq 2$. Then

$$
(x+n)^{2} x^{k}-x^{2} x^{k}=x^{k}\left(2 n x+n^{2}\right)=2 n x^{k+1}+n^{2} x^{k} \in V
$$

for all $n \in \mathbb{Z}$. Thus $x^{k+1} \in V$. By induction, $V=\mathbb{C}[x]$ and $\mathfrak{g}_{V}=\mathfrak{g}_{\langle 1\rangle}=\mathfrak{g}$. 
Case 2: $r=1, p(x)=x+a$. Since

$$
p(x) q(x)-p(x) q(x+m)=-p(x)\left(q^{\prime}(x) m+\frac{q^{(2)}(x)}{2 !} m^{2}+\cdots+\frac{q^{(s)}(x)}{s !} m^{s}\right) \in V
$$

for all $m \in \mathbb{Z}$, we have

Thus

$$
(x+a) q^{\prime}(x), \ldots,(x+a) \frac{q^{(s)}(x)}{s !} \in V
$$

$$
x+a,(x+a) x, \ldots,(x+a) x^{s-1} \in V,
$$

where $s \geqq 2$. Assume that $(x+a),(x+a) x, \ldots,(x+a) x^{k} \in V$ for some $k \geqq 1$. Then

$$
\begin{gathered}
(x+a+n)(x+n)(x+a) x^{k}-(x+a) x(x+a) x^{k} \\
=(x+a) x^{k}(2 n x+n(a+n)) \in V
\end{gathered}
$$

for all $n$. Hence $(x+a) x^{k+1} \in V$. By induction, $\langle x+a\rangle \subseteq V$. Note that $\langle x+a\rangle$ has codimension 1 in $\mathbb{C}[x]$, so $\langle x+a\rangle=V$ and $\mathfrak{g}_{V}=\mathfrak{g}_{\langle x+a\rangle}$.

Case 3: $r \geqq 2$. For any $n \in \mathbb{Z}$, since

$$
\begin{aligned}
& p(x+n) p(x)-p(x) p(x) \\
& \quad=p(x)\left(p^{\prime}(x) n+\frac{p^{(2)}(x)}{2 !} n^{2}+\cdots+\frac{p^{(r)}(x)}{r !} n^{r}\right) \in V,
\end{aligned}
$$

we have

$$
p(x), x p(x), \ldots, x^{r-1} p(x) \in V
$$

where $r \geqq 2$. Assume that

$$
p(x), x p(x), \ldots, x^{k} p(x) \in V \quad \text { for some } k \geqq r-1 \geqq 1 .
$$

Since

we have

$$
1 \leqq k-r+2 \leqq k
$$

and

$$
r(x):=x^{k-r+2} p(x) \in V \text {, }
$$

But

$$
\operatorname{deg}(r(x))=k+2 \text {. }
$$

$$
\begin{aligned}
& r(x+n) p(x)-r(x) p(x) \\
& \quad=p(x)\left(r^{\prime}(x) n+\frac{r^{(2)}(x)}{2 !} n^{2}+\cdots+\frac{r^{(k+2)}(x)}{(k+2) !} n^{k+2}\right) \in V
\end{aligned}
$$

for all $n \in \mathbb{Z}$, so

$$
p(x), x p(x), \ldots, x^{k+1} p(x) \in V
$$

By induction, $\langle p(x)\rangle \subseteq V$. If there exists $h(x) \in V \backslash\langle p(x)\rangle$, then by the choice of $p(x)$,

$$
\operatorname{deg}(h(x)) \geqq \operatorname{deg}(p(x)) \geqq r
$$


Assume that $\operatorname{deg}(h(x))=l$ and define $h_{1}(x):=h(x)-x^{l-r} p(x)$. Then $h_{1}(x) \in$ $V \backslash\langle p(x)\rangle$ and $\operatorname{deg}\left(h_{1}(x)\right)<\operatorname{deg}(h(x))$. Inductively, there exists a $h^{*}(x) \in V \backslash\langle p(x)\rangle$ such that $\operatorname{deg}\left(h^{*}(x)\right)<\operatorname{deg}(p(x))$. This is a contradiction. So we have $V=\langle p(x)\rangle$ and complete the proof.

\section{Graded Automorphism Group of $\mathbb{C}\left[t, t^{-1}, \frac{d}{d t}\right]$}

We had seen in Sect. 1 that $\mathfrak{g}=\mathbb{C}\left[t, t^{-1}, \frac{d}{d t}\right]$ is $\mathbb{Z}$-graded as $\mathfrak{g}=\sum_{m \in \mathbb{Z}} \mathfrak{g}_{m}$, $\mathfrak{g}_{m}=\sum_{r \in \mathbb{Z}_{\geq 0}} \mathbb{C} d_{m}^{r}$. Let $\phi \in A u t(\mathfrak{g})$, the automorphism group of $\mathfrak{g}$. If $\phi\left(\mathfrak{g}_{m}\right)=\mathfrak{g}_{m}$ for all $m \in \mathbb{Z}$, we say $\phi$ is a graded automorphism of $\mathfrak{g}$. Let

$$
A u t_{G}(\mathfrak{g})=\{\phi \in A u t(\mathfrak{g}) \mid \phi \text { is graded }\} \text {. }
$$

Then $A u t_{G}(\mathfrak{g})$ is a subgroup of $A u t(\mathfrak{g})$. In this section, we determine $A u t_{G}(\mathfrak{g})$. First we prove the following.

Proposition 1. As a Lie algebra, $\mathfrak{g}$ is generated by $d_{-1}^{0}, d_{1}^{0}, d_{0}^{2}$; that is $\mathfrak{g}=\left\langle d_{-1}^{0}, d_{1}^{0}, d_{0}^{2}\right\rangle$.

Proof. Let $\mathrm{g}^{\prime}:=\left\langle d_{-1}^{0}, d_{1}^{0}, d_{0}^{2}\right\rangle$. Since $\left[d_{0}^{2}, d_{1}^{0}\right]=2 d_{1}^{1}+d_{1}^{0} \in \mathrm{g}$, we have

$$
d_{1}^{1} \in \mathfrak{g}^{\prime} \text {. }
$$

Similarly, we have

Thus

$$
d_{-1}^{1} \in \mathfrak{g}^{\prime} .
$$

$$
d_{0}^{1}=\frac{1}{2}\left[d_{-1}^{1}, d_{1}^{1}\right] \in \mathfrak{g}^{\prime} .
$$

Now $\left[d_{0}^{2}, d_{1}^{1}\right]=2 d_{1}^{2}+d_{1}^{1} \in \mathfrak{g}^{\prime}$ implies that $d_{1}^{2} \in \mathfrak{g}^{\prime}$. Then $\left[d_{1}^{1}, d_{1}^{0}\right]=d_{2}^{0} \in \mathfrak{g}^{\prime}$ and $\left[d_{0}^{2}, d_{1}^{0}\right]=2 d_{2}^{1}+d_{2}^{0} \in \mathfrak{g}^{\prime}$ imply that

$$
d_{2}^{1} \in \mathfrak{g}^{\prime}
$$

Similarly,

$$
d_{-2}^{1} \in \mathfrak{g}^{\prime} .
$$

Since $\mathfrak{g}\{x\}$ is generated by $\left\{d_{-2}^{1}, d_{-1}^{1}, d_{0}^{1}, d_{1}^{1}, d_{2}^{1}\right\}$,

Then

$$
\mathfrak{g}\{x\} \subseteq \mathfrak{g}^{\prime}
$$

$$
d_{n+1}^{0}=\left[d_{n}^{1}, d_{1}^{0}\right] \in \mathfrak{g}^{\prime}
$$

for all $n \in \mathbb{Z}$. Finally from $\left[d_{0}^{2}, d_{n}^{1}\right]=2 n d_{n}^{2}+n^{2} d_{n}^{1} \in \mathfrak{g}^{\prime}$ for all $n \in \mathbb{Z}$, we obtain

$$
d_{n}^{2} \in \mathfrak{g}^{\prime}
$$

for all $n \in \mathbb{Z}$. In summary, we proved that

$$
d_{m}\left(x^{k}\right) \in \mathfrak{g}^{\prime} \quad \forall m \in \mathbb{Z}, k=0,1,2 .
$$

Now assume that $d_{m}\left(x^{k}\right) \in \mathfrak{g}^{\prime} \forall m \in \mathbb{Z}, 0 \leqq k \leqq l$. Then

$$
\left[d_{m}^{2}, d_{n}\left(x^{l}\right)\right]=d_{m+n}\left((x+n)^{2} x^{l}-x^{2}(x+m)^{l}\right) \in \mathfrak{g}^{\prime}
$$

implies that $d_{m}\left(x^{l+1}\right) \in \mathfrak{g}^{\prime}$ for all $m \in \mathbb{Z}$. By induction, we get $\mathfrak{g}^{\prime}=\mathfrak{g}$. 
Definition. Let $\beta \in \mathbb{C}$ and define $\psi_{\beta}: \mathfrak{g} \rightarrow \mathfrak{g}$ to be the unique linear map such that $\psi_{\beta}\left(d_{m}(p(x))\right)=d_{m}(p(x+\beta))$ for all $m \in \mathbb{Z}$ and $p(x) \in \mathbb{C}[x]$.

Let $a \in \mathbb{C}^{*}$ and define $\phi_{a}: \mathfrak{g} \rightarrow \mathfrak{g}$ to be the unique linear map such that $\phi_{a}\left(d_{m}(p(x))\right)=a^{m} d_{m}(p(x))$ for all $m \in \mathbb{Z}$ and $p(x) \in \mathbb{C}[x]$.

Let $\tau: \mathfrak{g} \rightarrow \mathfrak{g}$ be the linear map such that

$$
\tau\left(d_{m}(p(x))\right)=(-1)^{m-1} d_{m}(p(-x-m))
$$

for all $m \in \mathbb{Z}$ and $p(x) \in \mathbb{C}[x]$.

Proposition 2. Let $\Psi=\left\{\psi_{\beta} \mid \beta \in \mathbb{C}\right\}$ and let $\Phi=\left\{\phi_{a} \mid a \in \mathbb{C}^{*}\right\}$. Then Aut $(\mathfrak{g})=$ $(\langle\tau\rangle \bowtie \Psi) \times \Phi \cong\left(\mathbb{Z}_{2} \bowtie(\mathbb{C},+)\right) \times\left(\mathbb{C}^{*}, \cdot\right)$, where $\tau^{2}=1$.

Proof. Since

$$
\begin{aligned}
& {\left[\psi_{\beta}\left(d_{m}(p(x))\right), \psi_{\beta}\left(d_{n}(p(x))\right)\right]} \\
& \quad=\left[d_{m}(p(x+\beta)), d_{n}(q(x+\beta))\right] \\
& \quad=d_{m+n}(p(x+n+\beta) q(x+\beta)-p(x+\beta) q(x+m+\beta)) \\
& \quad=\psi_{\beta}\left(\left[d_{m}(p(x)), d_{n}(q(x))\right]\right),
\end{aligned}
$$

$\psi_{\beta} \in A u t_{G}(\mathfrak{g})$ for all $\beta \in \mathbb{C}$. It is easy to see from the definition that $\phi_{a} \in A u t_{G}(\mathfrak{g})$ for all $a \in \mathbb{C}^{*}, \psi_{\alpha} \psi_{\beta}=\psi_{\alpha+\beta}, \phi_{a} \phi_{b}=\phi_{a b}$, and $\phi_{a} \psi_{\beta}=\psi_{\beta} \phi_{a}$. It is also easy to verify that $\tau \in A u t_{G}(\mathfrak{g}), \quad \tau^{2}=1$, and $\tau \phi_{a}=\phi_{a} \tau, \quad \tau \psi_{\beta} \tau^{-1}=\psi_{-\beta}$. Hence $(\langle\tau\rangle \ltimes \Psi) \times \Phi \cong$ $\left(\mathbb{Z}_{2} \bowtie(\mathbb{C},+)\right) \times\left(\mathbb{C}^{*}, \cdot\right)$ is a subgroup of $A u t_{G}(\mathfrak{g})$.

Now suppose $\psi \in A u t_{G}(\mathrm{~g})$. We show that $\psi \in(\langle\tau\rangle \triangleright\langle\Psi) \times \Phi$. Assume that $\psi\left(d_{m}^{0}\right)=\sum_{i=0}^{l_{m}} a_{m, i} d_{m}^{i}$, where $m \in \mathbb{Z}$, and $a_{m, l_{m}} \neq 0$. Fix $m \in \mathbb{Z}$. For any $n \in \mathbb{Z}$,

$$
\begin{aligned}
0 & =\psi\left[d_{m}^{0}, d_{n}^{0}\right] \\
& =\left[\psi\left(d_{m}^{0}\right), \psi\left(d_{n}^{0}\right)\right] \\
& =a_{m, l_{m}} a_{n, l_{n}}\left[d_{m}^{l_{m}}, d_{n}^{l_{n}}\right]+\cdots \\
& =a_{m, l_{m}} a_{n, l_{n}} d_{m+n}\left((x+n)^{l_{m}} x^{l_{n}}-x^{l_{m}}(x+m)^{l_{n}}\right)+\cdots .
\end{aligned}
$$

If $l_{m}>0$, then for $n \gg 0, d_{m+n}\left((x+n)^{l_{m}} x^{l_{n}}-x^{l_{m}}(x+m)^{l_{n}}\right) \neq 0$. So we must have $l_{m}=0$, i.e. $\psi\left(d_{m}^{0}\right)=a_{m} d_{m}^{0}$ for some $a_{m} \in \mathbb{C}^{*}$.

Assume that $\psi\left(d_{m}^{1}\right)=d_{m}\left(p_{m}(x)\right)$. Comparing

$$
\psi\left[d_{m}^{1}, d_{n}^{0}\right]=\psi\left(n d_{m+n}^{0}\right)=n a_{m+n} d_{m+n}^{0}
$$

with

$$
\begin{aligned}
{\left[\psi\left(d_{m}^{1}\right), \psi\left(d_{n}^{0}\right)\right] } & =\left[d_{m}\left(p_{m}(x)\right), a_{n} d_{n}^{0}\right] \\
& =a_{n} d_{m+n}\left(p_{m}(x+n)-p_{m}(x)\right) \\
& =a_{n} d_{m+n}\left(p_{m}^{\prime}(x) n+\frac{p_{m}^{(2)}(x)}{2 !} n^{2}+\cdots\right)
\end{aligned}
$$

we obtain

$$
\operatorname{deg}\left(p_{m}^{\prime}(x)\right)=0,
$$


and

$$
p_{m}(x)=b_{m} x+c_{m}
$$

for some $b_{m}, c_{m} \in \mathbb{C}$. Thus

$$
\psi\left(d_{m}^{1}\right)=b_{m} d_{m}^{1}+c_{m} d_{m}^{0}
$$

Moreover, from $\psi\left[d_{m}^{1}, d_{n}^{0}\right]=\left[\psi\left(d_{m}^{1}\right), \psi\left(d_{n}^{0}\right)\right]$, we have

$$
n a_{m+n}=n a_{n} b_{m} \quad \forall m, n \in \mathbb{Z} .
$$

Assume that $\psi\left(d_{0}^{2}\right)=d_{0}(q(x))$. Then

$$
\begin{aligned}
\psi\left[d_{0}^{2}, d_{n}^{0}\right] & =\psi\left(d_{n}\left(2 n x+n^{2}\right)\right) \\
& =2 n \psi\left(d_{n}^{1}\right)+n^{2} \psi\left(d_{n}^{0}\right) \\
& =2 n b_{n} d_{n}^{1}+\left(2 n c_{n}+n^{2} a_{n}\right) d_{n}^{0},
\end{aligned}
$$

and

$$
\begin{aligned}
{\left[\psi\left(d_{0}^{2}\right), \psi\left(d_{n}^{0}\right)\right] } & =\left[d_{0}(q(x)), a_{n} d_{n}^{0}\right] \\
& =a_{n} d_{n}(q(x+n)-q(x)) \\
& =a_{n} d_{n}\left(q^{\prime}(x) n+\frac{q^{(2)}(x)}{2 !} n^{2}+\cdots\right)
\end{aligned}
$$

for all $n \in \mathbb{Z}$. These imply that $\operatorname{deg}\left(q^{\prime}(x)\right)=1$, and $q(x)=\beta_{2} x^{2}+\beta_{1} x+\beta_{0}$ for some $\beta_{2}, \beta_{1}, \beta_{0} \in \mathbb{C}$, where $\beta_{2} \neq 0$. From $\psi\left[d_{0}^{2}, d_{n}^{0}\right]=\left[\psi\left(d_{0}^{2}\right), \psi\left(d_{n}^{0}\right)\right]$, we get

$$
\left\{\begin{array}{l}
2 n b_{n}=2 n \beta_{2} a_{n} \\
2 n c_{n}+n^{2} a_{n}=a_{n}\left(\beta_{1} n+\beta_{2} n^{2}\right)
\end{array}\right.
$$

for all $n \in \mathbb{Z}$.

With $m=0$ and $n=1$ in (1), and by (2), we get

$$
\left\{\begin{array}{l}
b_{0}=1 \\
b_{n}=\beta_{2} a_{n} \quad n \neq 0,
\end{array}\right.
$$

and

$$
c_{n}=\frac{a_{n}}{2}\left(\beta_{1}+\left(\beta_{2}-1\right) n\right) \text { if } n \neq 0
$$

Hence

$$
\left\{\begin{array}{l}
\psi\left(d_{n}^{1}\right)=a_{n}\left(\beta_{2} d_{n}^{1}+\frac{\beta_{1}+\left(\beta_{2}-1\right) n}{2} d_{n}^{0}\right) \text { if } n \neq 0 \\
\psi\left(d_{0}^{1}\right)=d_{0}^{1}+c_{0} d_{0}^{0}
\end{array}\right.
$$

Then from

$$
\psi\left[d_{n}^{1}, d_{-n}^{1}\right]=-2 n \psi\left(d_{0}^{1}\right)=-2 n\left(d_{0}^{1}+c_{0} d_{0}^{0}\right)
$$


and

$\left[\psi\left(d_{n}^{1}\right), \psi\left(d_{-n}^{1}\right)\right]$

$$
\begin{aligned}
& =\left[a_{n}\left(\beta_{2} d_{n}^{1}+\frac{\beta_{1}+\left(\beta_{2}-1\right) n}{2} d_{n}^{0}\right), a_{-n}\left(\beta_{2} d_{-n}^{1}+\frac{\beta_{1}-\left(\beta_{2}-1\right) n}{2} d_{-n}^{0}\right)\right] \\
& =-2 n \beta_{2}^{2} a_{n} a_{-n} d_{0}^{1}-n a_{n} a_{-n} \beta_{1} \beta_{2} d_{0}^{0},
\end{aligned}
$$

we get $a_{n} a_{-n} \beta_{2}^{2}=1$, and $c_{0}=\frac{a_{n} a_{-n}}{2} \beta_{1} \beta_{2}$ for all $n \neq 0$. Hence

$$
\left\{\begin{array}{l}
c_{0}=\frac{\beta_{1}}{2 \beta_{2}} \\
c_{n}=\frac{a_{n}}{2}\left(\beta_{1}+\left(\beta_{2}-1\right) n\right) .
\end{array}\right.
$$

From (1) and (3), and using $a_{1} a_{-1} \beta_{2}^{2}=1$, we have

$$
a_{m+1}=a_{1} a_{m} \beta_{2} \quad \forall m \in \mathbb{Z} .
$$

By induction on $m$, we get

$$
a_{m}=\beta_{2}^{m-1} a^{m} \quad \forall m \in \mathbb{Z},
$$

where $a=a_{1}$. Then from (3) and (4),

$$
\left\{\begin{array}{l}
b_{m}=\beta_{2}^{m-1} a^{m} \beta_{2} \\
c_{m}=\beta_{2}^{m-1} a^{m} \frac{\beta_{1}+\left(\beta_{2}-1\right) m}{2}
\end{array}\right.
$$

for all $m \in \mathbb{Z}$.

In summary, we have proved that

$$
\left\{\begin{array}{l}
\psi\left(d_{n}^{0}\right)=\beta_{2}^{n-1} a^{n} d_{n}^{0} \\
\psi\left(d_{n}^{1}\right)=\beta_{2}^{n-1} a^{n} d_{n}\left(\beta_{2} x+\frac{\beta_{1}+\left(\beta_{2}-1\right) n}{2}\right)
\end{array}\right.
$$

for all $n \in \mathbb{Z}$. Assume that

$$
\psi\left(d_{n}^{r}\right)=\beta_{2}^{n-1} a^{n} d_{n}\left(\left(\beta_{2} x+\frac{\beta_{1}+\left(\beta_{2}-1\right) n}{2}\right)^{r}\right)
$$

for all $n \neq 0$. From $\psi\left[d_{n}^{r}, d_{0}^{2}\right]=\left[\psi\left(d_{n}^{r}\right), \psi\left(d_{0}^{2}\right)\right]$, we get

$$
\psi\left(d_{n}^{r+1}\right)=\beta_{2}^{n-1} a^{n} d_{n}\left(\left(\beta_{2} x+\frac{\beta_{1}+\left(\beta_{2}-1\right) n}{2}\right)^{r+1}\right)
$$

for all $n \neq 0$. So by induction on $r$, we obtain

$$
\psi\left(d_{n}^{r}\right)=\beta_{2}^{n-1} a^{n} d_{n}\left(\left(\beta_{2} x+\frac{\beta_{1}+\left(\beta_{2}-1\right) n}{2}\right)^{r}\right)
$$

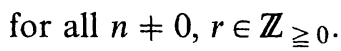

Since

$$
\psi\left[d_{n}^{3}, d_{-n}^{0}\right]=-3 n \psi\left(d_{0}^{2}\right)+3 n^{2} \psi\left(d_{0}^{1}\right)-n^{3} \psi\left(d_{0}^{0}\right)
$$


and

$\left[\psi\left(d_{n}^{3}\right), \psi\left(d_{-n}^{0}\right)\right]$

$$
\begin{aligned}
= & {\left[\beta_{2}^{n-1} a^{n} d_{n}\left(\left(\beta_{2} x+\frac{\beta_{1}+\left(\beta_{2}-1\right) n}{2}\right)^{3}\right), \beta_{2}^{-n-1} a^{-n} d_{-n}(1)\right] } \\
= & \beta_{2}^{-2} d_{0}\left(\left(\beta_{2} x+\frac{\beta_{1}+\left(\beta_{2}-1\right) n}{2}-\beta_{2} n\right)^{3}-\left(\beta_{2} x+\frac{\beta_{1}+\left(\beta_{2}-1\right) n}{2}\right)^{3}\right) \\
= & \beta_{2}^{-2} d_{0}\left(3\left(\beta_{2} x+\frac{\beta_{1}+\left(\beta_{2}-1\right) n}{2}\right)^{2}\left(-\beta_{2} n\right)\right. \\
& \left.+3\left(\beta_{2} x+\frac{\beta_{1}+\left(\beta_{2}-1\right) n}{2}\right)\left(-\beta_{2} n\right)^{2}+\left(-\beta_{2} n\right)^{3}\right)
\end{aligned}
$$

and

$$
\psi\left[d_{n}^{3}, d_{-n}^{0}\right]=\left[\psi\left(d_{n}^{3}\right), \psi\left(d_{-n}^{0}\right)\right]
$$

for arbitrary $n \neq 0$, using (7) and comparing the coefficients of $n^{3}$ and $n$, we get

$$
\beta_{2}^{2}=1
$$

and

$$
\beta_{0}=\beta_{2}^{-1}\left(\frac{\beta_{1}}{2}\right)^{2}
$$

If $\beta_{2}=1$, then (7) and $\beta_{0}=\left(\frac{\beta_{1}}{2}\right)^{2}$ give us

$$
\left\{\begin{array}{l}
\psi\left(d_{1}^{0}\right)=a d_{1}^{0}=\phi_{a} \psi_{\frac{\beta_{1}}{2}}\left(d_{1}^{0}\right) \\
\psi\left(d_{-1}^{0}\right)=a^{-1} d_{-1}^{0}=\phi_{a} \psi_{\frac{\beta_{1}}{2}}\left(d_{-1}^{0}\right) \\
\psi\left(d_{0}^{2}\right)=d_{0}\left(\left(x+\frac{\beta_{1}}{2}\right)^{2}\right)=\phi_{a} \psi_{\frac{\beta_{1}}{2}}\left(d_{0}^{2}\right) .
\end{array}\right.
$$

If $\beta_{2}=-1$, then (7) and $\beta_{0}=\left(\frac{\beta_{1}}{2}\right)^{2}$ give us

$$
\left\{\begin{array}{l}
\psi\left(d_{1}^{0}\right)=a d_{1}^{0}=\phi_{a} \tau \psi_{\frac{\beta_{1}}{2}}\left(d_{1}^{0}\right) \\
\psi\left(d_{-1}^{0}\right)=a^{-1} d_{-1}^{0}=\phi_{a} \tau \psi_{\frac{\beta_{1}}{2}}\left(d_{-1}^{0}\right) \\
\psi\left(d_{0}^{2}\right)=-d_{0}\left(\left(-x+\frac{\beta_{1}}{2}\right)^{2}\right)=\phi_{a} \tau \psi_{\frac{\beta_{1}}{2}}\left(d_{0}^{2}\right) .
\end{array}\right.
$$

Since by Proposition $1, d_{0}^{2}, d_{1}^{0}$ and $d_{-1}^{0}$ generate $\mathrm{g}$, so in both cases above $\psi \in\left\langle\tau, \psi_{\beta}, \phi_{a} \mid \beta \in \mathbb{C}, a \in \mathbb{C}^{*}\right\rangle$.

Hence

$$
A u t_{G}(\mathrm{~g})=(\langle\tau\rangle \ltimes \Psi) \times \Phi .
$$




\section{Structure of Homogeneous Lie Subalgebras of $\mathbb{C}\left[t, t^{-1}, \frac{d}{d t}\right]$}

It is obvious that $\mathfrak{g}\{0\}$ is abelian, $\mathfrak{g}\{x+\alpha\}$ is simple, and $\mathfrak{g}\{0,1\}=\mathfrak{g}\{x\} \triangleright<\mathfrak{g}\{0\}$. In this section, we discuss the structure of $\mathfrak{g}_{\langle p(x)\rangle}$ further.

Proposition 1. Let $p(x), q(x) \in \mathbb{C}[x]$. Then

(i) $\mathfrak{g}_{\langle q(x)\rangle}$ is a Lie subalgebra of $\mathfrak{g}_{\langle p(x)\rangle}$ iff $p(x) \mid q(x)$.

(ii) $\mathfrak{g}_{\langle q(x)\rangle}$ is an ideal of $\mathfrak{g}_{\langle p(x)\rangle}$ iff $\langle p(x)\rangle=\langle q(x)\rangle$.

Proof. (i) is clear.

(ii) Suppose that $\mathfrak{g}_{\langle q(x)\rangle}$ is an ideal of $\mathfrak{g}_{\langle p(x)\rangle}$. Then

$$
\left[d_{m}(p(x)), d_{n}(q(x))\right]=d_{m+n}(p(x+n) q(x)-p(x) q(x+m)) \in \mathfrak{g}_{\langle q(x)\rangle} .
$$

Hence

$$
q(x) \mid p(x) q(x+m) \quad \forall m \in \mathbb{Z} .
$$

Assume that $q(x)=p(x) r(x)$. Then

$$
r(x) \mid q(x+m) \quad \forall m \in \mathbb{Z} .
$$

So $r(x)$ must be a constant and $\langle p(x)\rangle=\langle q(x)\rangle$.

Proposition 2. Let

$$
p(x), q(x) \in \mathbb{C}[x], \quad \text { where g.c.d. }(p(x), q(x))=1,
$$

and let

$$
r(x)=p(x) q(x)
$$

Then

$$
\mathfrak{g}_{\langle r(x)\rangle}=\mathfrak{g}_{\langle p(x)\rangle} \cap \mathfrak{g}_{\langle q(x)\rangle} .
$$

In particular, if

$$
r(x)=\prod_{i=1}^{r}\left(x+\alpha_{i}\right)^{k_{i}},
$$

where $\alpha_{1}, \ldots, \alpha_{r}$ are distinct, then

$$
\mathfrak{g}_{\langle r(x)\rangle}=\bigcap_{i=1}^{r} \mathfrak{g}_{\left\langle\left(x+\alpha_{i}\right)^{k_{i}}\right\rangle} .
$$

Proof. Let $X \in \mathfrak{g}_{\langle p(x)\rangle} \cap \mathfrak{g}_{\langle q(x)\rangle}$. Then

$$
X=\sum_{m} d_{m}\left(p(x) h_{m}(x)\right)=\sum_{m} d_{m}\left(q(x) k_{m}(x)\right),
$$

where $h_{m}(x), \quad k_{m}(x) \in \mathbb{C}[x]$. So $p(x) h_{m}(x)=q(x) k_{m}(x)$ for all $m$. But g.c.d. $(p(x), q(x))=1$, so $p(x) \mid k_{m}(x)$ for all. Assume that $k_{m}(x)=p(x) k_{m}^{*}(x)$, for some $k_{m}^{*}(x) \in \mathbb{C}[x]$. Then $X=\sum_{m} d_{m}\left(q(x) p(x) k_{m}^{*}(x)\right) \in \mathfrak{g}_{\langle r(x)\rangle}$.

It is clear that $\mathbb{C} d_{0}$ is the centre of $\mathfrak{g}$. Moreover, we have the following:

Proposition 3. $\mathbb{C} d_{0}^{0}$ is the only proper ideal of $\mathfrak{g}$ and hence $\mathfrak{g} / \mathbb{C} d_{0}^{0}$ is simple. 
Proof. Let $0 \neq I \triangleleft g$ be an ideal. From $\left[d_{0}^{1}, I\right] \subseteq I$, we see that $I=\sum_{m \in \mathbb{Z}} I_{m}$, where $I_{m}=I \cap g_{m}$. Suppose that $I \backslash \mathbb{C} d_{0}^{0}$ is not empty and $0 \neq d_{m}(p(x)) \in I \backslash \mathbb{C} d_{0}^{0}$ such that $\operatorname{deg}(p(x))$ is minimal. If $\operatorname{deg}(p(x))>0$, then

$$
\left[d_{m}\left(p(x), d_{n}^{0}\right]=d_{m+n}(p(x+n)-p(x)) \in I\right.
$$

and

$$
\operatorname{deg}(p(x+n)-p(x))<\operatorname{deg}(p(x)) .
$$

This is a contradiction. So $p(x) \in \mathbb{C}$, and $d_{m}^{0} \in I \backslash \mathbb{C} d_{0}^{0}$. This implies that

$$
\left[d_{n}^{1}, d_{m}^{0}\right]=m d_{m+n}^{0} \in I \quad \forall n \in \mathbb{Z} .
$$

Hence $g\{0\} \subseteq I$. Note that $\left[d_{m}\left(x^{r}\right), d_{n}^{0}\right]=d_{m+n}\left((x+n)^{r}-x^{r}\right) \in I$ for all $m, n \in \mathbb{Z}$, $r \in \mathbb{Z} \geqq 0$. By induction on $r$, we see that $d_{m}\left(x^{r}\right) \in I, \forall m \in \mathbb{Z}, r \in \mathbb{Z} \geqq 0$. So $I=\mathfrak{g}$.

Proposition 4. For any $\alpha \in \mathbb{C}, \mathfrak{g}_{\langle x+\alpha\rangle}$ is simple.

Proof. By Sect. 2, Proposition 2, we need only to show that $\mathfrak{g}_{\langle x\rangle}$ is simple. Let $0 \neq I \triangleleft \mathfrak{g}_{\langle x\rangle}$ be an ideal. Then $I=\sum_{m \in \mathbb{Z}} I_{m}$, where $I_{m}=I \cap g_{m}$. Let $d_{n}(x p(x)) \in I$ and $\operatorname{deg}(p(x))$ be minimal. Since

$$
\left[d_{m}(x), d_{n}(x p(x))\right]=d_{m+n}((x+n) x p(x)-x(x+m) p(x+m)) \in I
$$

for all $m \in \mathbb{Z}$, we have

$$
\operatorname{deg}((x+n) p(x)-(x+m) p(x+m)) \geqq \operatorname{deg}(p(x))
$$

for all $m \in \mathbb{Z}$. On the other hand,

$$
\operatorname{deg}((x+n) p(x)-(x+m) p(x+m)) \leqq \operatorname{deg}(p(x)) .
$$

So they must be equal. Thus for any $m \in \mathbb{Z}$, there exists $p_{m}(x)$ with $\operatorname{deg}\left(p_{m}(x)\right)=\operatorname{deg}(p(x))$ and $d_{m}\left(x p_{m}(x)\right) \in I$. We may assume that the coefficient of the highest term of $p_{m}(x)$ is 1 . Then

$$
\left[d_{m}(x), d_{n}\left(x p_{n}(x)\right)\right]=d_{m+n}\left((x+n) x p_{n}(x)-x(x+m) p_{n}(x+m)\right) \in I
$$

for all $m, n \in \mathbb{Z}$. Fix $m \neq 0$ and choose $n=m(1+\operatorname{deg}(p(x)))$. Then

$$
\begin{aligned}
(x & +n) p_{n}(x)-(x+m) p_{n}(x+m) \\
& =(x+n) p_{n}(x)-(x+m)\left(p_{n}(x)+p_{n}^{\prime}(x) m+\cdots\right) \\
& =(n-m) p_{n}(x)-x p_{n}(x)^{\prime} m+\text { lower terms } .
\end{aligned}
$$

So

$$
\operatorname{deg}\left((x+n) p_{n}(x)-(x+m) p_{n}(x+m)\right)<\operatorname{deg}(p(x))
$$

unless $\operatorname{deg}(p(x))=0$. So $d_{m}(x) \in I$ for all $m \in \mathbb{Z}$.

Now from $\left[d_{m}(x), d_{n}\left(x^{r+1}\right)\right] \in I \quad \forall r \in \mathbb{Z}_{\geqq 0}$, and by induction on $r$, we get $I=\mathfrak{g}_{\langle x\rangle}$.

Proposition 5. For any $p(x) \in \mathbb{C}[x], \mathfrak{g}_{\langle p(x)\rangle}$ is indecomposable.

Proof. If $\operatorname{deg}(p(x)) \leqq 1$, this follows from Proposition 3 and 4 . Now assume that $\operatorname{deg}(p(x)) \geqq 2$. Suppose $g_{\langle p(x)\rangle}=U_{1} \oplus U_{2}$, where $U_{1}, U_{2}$ are ideals of $g_{\langle p(x)\rangle}$ and $U_{1} \cap U_{2}=0$ and both $U_{1}$ and $U_{2}$ are non-zero. Let

$$
0 \neq y=d_{j_{1}}\left(q_{1}(x)\right)+d_{j_{2}}\left(q_{2}(x)\right)+\cdots+d_{j_{k}}\left(q_{k}(x)\right) \in U_{1},
$$


where $j_{1}<j_{2}<\cdots<j_{k}$ and $q_{1}(x), q_{2}(x), \ldots, q_{k}(x)$ are non-zero. Then for all $m \in \mathbb{Z}$,

$$
\begin{aligned}
y_{m}: & =\left[d_{m}(p(x)), y\right] \\
& =\left[d_{m}(p(x)), d_{j_{1}}\left(q_{1}(x)\right)\right]+\cdots \\
& =d_{m+j_{1}}\left(p\left(x+j_{1}\right) q_{1}(x)-p(x) q_{1}(x+m)\right)+\cdots \in U_{1} .
\end{aligned}
$$

Note that

$$
q_{1}^{(m)}:=p\left(x+j_{1}\right) q_{1}(x)-p(x) q_{1}(x+m) \neq 0
$$

if $m \gg 0$, and

$$
\operatorname{deg}\left(q_{1}^{(m)}(x)\right) \leqq \operatorname{deg}(p(x))+\operatorname{deg}\left(q_{1}(x)\right)
$$

for all $m \in \mathbb{Z}$.

Let

$$
0 \neq z=d_{i_{1}}\left(r_{1}(x)\right)+\cdots+d_{i_{s}}\left(r_{s}(x)\right) \in U_{2},
$$

where $i_{1}<\cdots<i_{2}$, and $r_{1}(x), \ldots, r_{s}(x)$ are non-zero. Then $\left[y_{m}, z\right]=0$ for all $m \in \mathbb{Z}$. This implies

$$
\begin{aligned}
& {\left[d_{m+j_{1}}\left(q_{1}^{(m)}(x)\right), d_{i_{1}}\left(r_{1}(x)\right)\right]} \\
& \quad=d_{m+j_{1}+i_{1}}\left(q_{1}^{(m)}\left(x+i_{1}\right) r_{1}(x)-q_{1}^{(m)}(x) r_{1}(x+m)\right) \\
& \quad=0
\end{aligned}
$$

for all $m \in \mathbb{Z}$. But

$$
\operatorname{deg}\left(q_{1}^{(m)}(x)\right) \leqq \operatorname{deg}(p(x))+\operatorname{deg}\left(q_{1}(x)\right)
$$

for all $m \in \mathbb{Z}$, so

$$
q_{1}^{(m)}\left(x+i_{1}\right) r_{1}(x)-q_{1}^{(m)}(x) r_{1}(x+m) \neq 0
$$

if $m \gg 0$. Hence

$$
d_{m+j_{1}+i_{1}}\left(q_{1}^{(m)}\left(x+i_{1}\right) r_{1}(x)-q_{1}^{(m)}(x) r_{1}(x+m)\right) \neq 0
$$

if $m \gg 0$. This is a contradiction.

\section{Central Extensions}

In this section we consider the 1-dimensional central extensions of the homogeneous differential operator Lie algebras and determine the universal central extension of $\mathfrak{g}\{0,1\}$.

$$
\begin{aligned}
& \text { Recall that } \mathfrak{g l}_{\infty}(\mathbb{C})=\sum_{i, j \in \mathbb{Z}} \mathbb{C} E_{i j} \text { with Lie bracket } \\
& {\left[E_{i j}, E_{k l}\right]=\delta_{j k} E_{i l}-\delta_{l i} E_{k j}}
\end{aligned}
$$

is a Lie-algebra, where $E_{i j}$ is the matrix with 1 in $i$-row and $j$-column, with 0 elsewhere. Define

$$
\alpha: \mathfrak{g l}_{\infty}(\mathbb{C}) \times \mathrm{gl}_{\infty}(\mathbb{C}) \rightarrow \mathbb{C}
$$


to be bilinear and

$$
\begin{cases}\alpha\left(E_{i j}, E_{j i}\right)=-\alpha\left(E_{j i}, E_{i j}\right)=1 & i \leqq 0, j \geqq 1 \\ \alpha\left(E_{i j}, E_{k l}\right)=0 & \text { otherwise }\end{cases}
$$

Then $\alpha$ is a 2-cocycle of the Lie algebra $\mathrm{gl}_{\infty}(\mathbb{C})$.

$\mathrm{Kac}$ and Raina [KR] defined the following infinite matrix Lie algebra:

$$
\overline{\mathfrak{a}_{\infty}}:=\left\{A=\left(a_{i j}\right)_{i, j \in \mathbb{Z}}\left|a_{i j} \in \mathbb{C}, a_{i j}=0 \quad \forall\right| i-j \mid \gg 0\right\},
$$

where $|i-j| \gg 0$ means that $|i-j|$ is sufficiently large. The Lie bracket of $\overline{a_{\infty}}$ is given by $[A, B]=A B-B A$, for all $A, B \in \overline{\mathfrak{a}_{\infty}}$, where $A B$ is the usual matrix multiplication. A matrix in $\overline{a_{\infty}}$ is a linear combination of matrices of the form

$$
\sum_{i \in \mathbb{Z}} \lambda_{i} E_{i+m, i}
$$

for $m \in \mathbb{Z}$.

The 2-cocycle $\alpha$ defined above can be extended to a 2-cocycle of the Lie algebra $\overline{a_{\infty}}$ as follows:

$$
\alpha\left(\sum_{i} \lambda_{i} E_{i+m, i}, \sum_{j} \mu_{j} E_{j+n, j}\right):=\sum_{i} \sum_{j} \lambda_{i} \mu_{j} \alpha\left(E_{i+m, i}, E_{j+n, j}\right) .
$$

Kac and Raina [KR] also defined the 1-dimensional central extension $\mathfrak{a}_{\infty}$ of $\overline{\mathfrak{a}_{\infty}}$.

$$
\mathfrak{a}_{\infty}:=\overline{\mathfrak{a}_{\infty}}+\mathbb{C} \phi
$$

and

$$
\begin{array}{ll}
{[x, y]:=x y-y x+\alpha(x, y) \phi,} & \\
{[x, \phi]:=0,} & \forall x, y \in \overline{\mathfrak{a}_{\infty}} .
\end{array}
$$

Let

$$
\pi: \mathfrak{a}_{\infty} \rightarrow \overline{\mathfrak{a}_{\infty}}
$$

be the canonical homomorphism given by $\pi(x)=x$, for all $x \in \overline{\mathfrak{a}_{\infty}}$, and $\pi(\phi)=0$. For any Lie subalgebra $\mathfrak{h}$ of $\overline{\mathfrak{a}_{\infty}}, \tilde{h}:=\pi^{-1}(\mathfrak{h})$ is a Lie subalgebra of $\mathfrak{a}_{\infty}$. In general, it is a 1-dimensional central extension of $\mathfrak{h}$.

In the following, we imbed $g$ as a Lie subalgebra of $\overline{\mathfrak{a}_{\infty}}$ and hence obtain the 1 -dimensional central extensions of $\mathfrak{g}$ and its Lie subalgebras.

Proposition 1. For any $\beta \in \mathbb{C}$, define

$$
i_{\beta}: \mathfrak{g} \rightarrow \overline{\mathfrak{a}_{\infty}}
$$

to be the linear map such that

$$
i_{\beta}\left(d_{m}^{r}\right)=\sum_{j \in \mathbb{Z}}(j+\beta)^{r} E_{j+m, j} .
$$

Then $i_{\beta}$ is an injective Lie algebra homomorphism. 
Proof.

$$
\begin{aligned}
{\left[i_{\beta}\left(d_{m}^{r}\right), i_{\beta}\left(d_{n}^{s}\right)\right]=} & \sum_{j} \sum_{i}(j+\beta)^{r}(i+\beta)^{s}\left[E_{j+m, j}, E_{i+n, i}\right] \\
= & \sum_{j} \sum_{i}(j+\beta)^{r}(i+\beta)^{s}\left(\delta_{j, i+n} E_{j+m, i}-\delta_{i, j+m} E_{i+n, j}\right) \\
= & \sum_{i}(i+n+\beta)^{r}(i+\beta)^{s} E_{i+m+n, i} \\
& -\sum_{j}(j+\beta)^{r}(j+m+\beta)^{s} E_{j+m+n, j} \\
= & \sum_{j}\left((j+n+\beta)^{r}(j+\beta)^{s}-(j+\beta)^{r}(j+\beta+m)^{s}\right) E_{j+m+n, j} \\
= & \sum_{j}\left(\sum_{k}\left(\left(\begin{array}{l}
r \\
k
\end{array}\right) n^{k}-\left(\begin{array}{l}
s \\
k
\end{array}\right) m^{k}\right)(j+\beta)^{r+s-k}\right) E_{j+m+n, j} \\
= & \sum_{k}\left(\left(\begin{array}{l}
r \\
k
\end{array}\right) n^{k}-\left(\begin{array}{l}
s \\
k
\end{array}\right) m^{k}\right) i_{\beta}\left(d_{m+n}^{r+s-k}\right) \\
= & i_{\beta}\left[d_{m}^{r}, d_{n}^{s}\right] .
\end{aligned}
$$

The injectivity of $i_{\beta}$ is clear.

Now if we identify $d_{m}^{r}$ with $\sum_{j} j^{r} E_{j+m, j}$, then for polynomials $p(x), q(x)$,

$$
\begin{aligned}
& \alpha\left(\sum_{j} p(j) E_{j+m, j}, \sum_{i} q(i) E_{i+n, i}\right) \\
& \quad=\sum_{j} \sum_{i} p(j) q(i) \alpha\left(E_{j+m, j}, E_{i+n, i}\right) \\
& \quad=\left(\sum_{1 \leqq j \leqq n} p(j) q(j-n)-\sum_{1 \leqq j \leqq n} p(j-m) q(j)\right) \delta_{m+n, 0} .
\end{aligned}
$$

So in $\tilde{\mathfrak{g}}=\pi^{-1}(\mathfrak{g})$, the Lie brackets are

$$
\begin{aligned}
{\left[d_{m}(p(x)), d_{n}(q(x))\right]=} & d_{m+n}(p(x+n) q(x)-p(x) q(x+m)) \\
& +\left(\sum_{1 \leqq j \leqq n} p(j) q(j-n)-\sum_{1 \leqq j \leqq n} p(j-m) q(j)\right) \delta_{m+n, 0} \phi . \\
{\left[d_{m}(p(x)), \phi\right]=} & 0 .
\end{aligned}
$$

Example 1:

Example 2:

$$
\begin{aligned}
\mathfrak{g}\{0\} & =\sum_{m \in \mathbb{Z}} \mathbb{C} d_{m}^{0}+\mathbb{C} \phi, \\
{\left[d_{m}^{0}, d_{n}^{0}\right] } & =-2 m \delta_{m+n, 0} \phi, \\
{\left[d_{m}^{0}, \phi\right] } & =0 .
\end{aligned}
$$

$$
\begin{aligned}
\mathfrak{g}\{x\} & =\sum_{m \in \mathbb{Z}} \mathbb{C} d_{m}^{1}+\mathbb{C} \phi, \\
{\left[d_{m}^{1}, d_{n}^{1}\right] } & =(n-m) d_{m+n}^{1}+\frac{m^{3}-m}{6} \delta_{m+n, 0} \phi, \\
{\left[d_{m}^{1}, \phi\right] } & =0 .
\end{aligned}
$$


We see that $\tilde{\mathfrak{g}}\{x\}$ is the Virasoro algebra, which is the universal central extension of $g\{x\}$.

Wanglai $\mathrm{Li}$ [Li] proved the following result: The 1-dimensional central extension of $g$ is unique up to a scalar multiple. In the rest of this section we determine the universal central extension of $\mathfrak{g}\{0,1\}$. Since $\mathfrak{g}\{0,1\}$ is perfect, its universal central extension exists.

Generally, if a Lie algebra $\mathfrak{h}$ is perfect, its universal central extension can be obtained as follows:

Consider $\wedge^{2} \mathfrak{h}$, the second exterior power of $\mathfrak{h}$. Let $I \subseteq \wedge^{2} \mathfrak{h}$ be the subspace spanned by all of the elements

$$
x \wedge[y, z]+y \wedge[z, x]+z \wedge[x, y]
$$

where $x, y, z \in \mathfrak{h}$. Let

$$
\pi: \mathfrak{h} \times \mathfrak{h} \rightarrow \frac{\wedge^{2} \mathfrak{h}}{I}
$$

be the canonical map

$$
(x, y) \mapsto x \pi y:=x \wedge y+I .
$$

Then $\pi$ is the "universal" 2-cocycle for $\mathfrak{h}$, and $\mathfrak{h} \oplus\left(\wedge^{2} \mathfrak{h} / I\right)$ acquires a Lie algebra structure through

$$
[x+u, y+v]=[x, y]+x \pi y \quad \text { where } x, y \in \mathfrak{h}, \quad u, v \in \frac{\wedge^{2} \mathfrak{h}}{I}
$$

Now

$$
\tilde{\mathfrak{h}}:=\left[\mathfrak{h} \oplus \frac{\wedge^{2} \mathfrak{h}}{I}, \mathfrak{h} \oplus \frac{\wedge^{2} \mathfrak{h}}{I}\right]
$$

together with the restriction to $\tilde{\mathfrak{h}}$ of the natural projection of $\mathfrak{h} \oplus\left(\wedge^{2} \mathfrak{h} / I\right)$ onto $\tilde{\mathfrak{h}}$ is the required universal central extension (see $[\mathrm{G}]$ or $[\mathrm{MoPi}]$ ).

In our case,

$$
\begin{aligned}
\mathfrak{g}\{0,1\}= & \sum_{m \in \mathbb{Z}} \mathbb{C} d_{m}^{1}+\sum_{m \in \mathbb{Z}} \mathbb{C} d_{m}^{0} . \\
& \frac{\wedge^{2} \mathfrak{g}\{0,1\}}{I}
\end{aligned}
$$

is spanned by

$$
\pi\left(d_{m}^{1}, d_{n}^{1}\right), \quad \pi\left(d_{m}^{1}, d_{n}^{0}\right), \quad \pi\left(d_{m}^{0}, d_{n}^{0}\right) \quad \forall m, n \in \mathbb{Z} .
$$

The 2-cocycle condition gives us

$$
\begin{aligned}
& \pi\left(d_{m}^{1},\left[d_{n}^{0}, d_{k}^{0}\right]\right)+\pi\left(d_{n}^{0},\left[d_{k}^{0}, d_{m}^{1}\right]\right)+\pi\left(d_{k}^{0},\left[d_{m}^{1}, d_{n}^{0}\right]\right) \\
& \quad=-k \pi\left(d_{n}^{0}, d_{m+k}^{0}\right)+n \pi\left(d_{k}^{0}, d_{m+n}^{0}\right) \\
& \quad=0
\end{aligned}
$$


for all $m n, k \in \mathbb{Z}$. Let $k=-m-n$ in (1). Then

$$
n \pi\left(d_{m+n}^{0}, d_{-m-n}^{0}\right)=(m+n) \pi\left(d_{n}^{0}, d_{-n}^{0}\right) .
$$

Thus

$$
\phi_{3}:=\frac{\pi\left(d_{n}^{0}, d_{-n}^{0}\right)}{n}
$$

is independent of $n, n \neq 0$. Let $k=0$ in (1). Then

$$
\pi\left(d_{n}^{0}, d_{n}^{0}\right)=0 \quad \forall n \in \mathbb{Z} .
$$

From this, setting $m+n=0$ in (1), we have

$$
\pi\left(d_{n}^{0}, d_{k-n}^{0}\right)=0 \quad \forall k \neq 0 .
$$

Combining these results, we get

$$
\pi\left(d_{m}^{0}, d_{n}^{0}\right)=m \delta_{m+n, 0} \phi_{3} .
$$

Again by 2-cocycle condition,

$$
\begin{aligned}
& \pi\left(d_{m}^{1},\left[d_{n}^{1}, d_{k}^{0}\right]\right)+\pi\left(d_{n}^{1},\left[d_{k}^{0}, d_{m}^{1}\right]\right)+\pi\left(d_{k}^{0},\left[d_{m}^{1}, d_{n}^{1}\right]\right) \\
& \quad=k \pi\left(d_{m}^{1}, d_{n+k}^{0}\right)-k \pi\left(d_{n}^{1}, d_{k+m}^{0}\right)+(n-m) \pi\left(d_{k}^{0}, d_{m+n}^{1}\right) \\
& \quad=0 .
\end{aligned}
$$

If $m=0$ in (3), then

$$
k \pi\left(d_{0}^{1}, d_{n+k}^{0}\right)=(k+n) \pi\left(d_{n}^{1}, d_{k}^{0}\right) .
$$

With $k=-m-n$ in (3), we get

$$
(m-n) \pi\left(d_{m+n}^{1}, d_{-m-n}^{0}\right)=(m+n)\left(\pi\left(d_{m}^{1}, d_{-m}^{0}\right)-\pi\left(d_{0}^{1}, d_{-n}^{0}\right)\right) .
$$

Replacing $m$ by $2 m$ in (4), we obtain

$$
\begin{aligned}
& (2 m-n) \pi\left(d_{2 m+n}^{1}, d_{-2 m-n}^{0}\right) \\
& =(2 m+n)\left(\pi\left(d_{2 m}^{1}, d_{-2 m}^{0}\right)-\pi\left(d_{n}^{1}, d_{-n}^{0}\right)\right) .
\end{aligned}
$$

Replacing $n$ by $m+n$ in (4), we obtain

$$
\begin{aligned}
& -n \pi\left(d_{2 m+n}^{1}, d_{-2 m-n}^{0}\right) \\
= & (2 m+n)\left(\pi\left(d_{m}^{1}, d_{-m}^{0}\right)-\pi\left(d_{m+n}^{1}, d_{-m-n}^{0}\right)\right) .
\end{aligned}
$$

Multiplying this by $m-n$ and replacing the $\pi\left(d_{m+n}^{1}, d_{-m-n}^{0}\right)$ term using (4), we obtain

$$
\begin{aligned}
& -n(m-n) \pi\left(d_{2 m+n}^{1}, d_{-2 m-n}^{0}\right) \\
= & -2 n(2 m+n) \pi\left(d_{m}^{1}, d_{-m}^{0}\right)+(2 m+n)(m+n) \pi\left(d_{n}^{1}, d_{-n}^{0}\right) .
\end{aligned}
$$


From (5) and (6), we get

$$
\begin{gathered}
-n(m-n)(2 m+n) \pi\left(d_{2 m}^{1}, d_{-2 m}^{0}\right)+2 n(2 m+n)(2 m-n) \pi\left(d_{0}^{1}, d_{-m}^{0}\right) \\
=(2 m+n) 2 m^{2} \pi\left(d_{n}^{1}, d_{-n}^{0}\right) .
\end{gathered}
$$

If $2 m+n \neq 0, m-n \neq 0, m \neq 0, n \neq 0$, then

$$
\frac{\pi\left(d_{2 m}^{1}, d_{-2 m}^{0}\right)-2 \pi\left(d_{m}^{1}, d_{-m}^{0}\right)}{m^{2}}=\frac{2 \pi\left(d_{m}^{1}, d_{-m}^{0}\right)}{m(m-n)}+\frac{2 \pi\left(d_{n}^{1}, d_{-n}^{0}\right)}{n(n-m)} .
$$

Thus

$$
\phi_{2}:=\frac{\pi\left(d_{2 m}^{1}, d_{-2 m}^{0}\right)-2 \pi\left(d_{m}^{1}, d_{-m}^{0}\right)}{m^{2}}
$$

is independent of $m, m \neq 0$.

Finally, since

$$
\pi\left(d_{m}^{1},\left[d_{n}^{1}, d_{k}^{1}\right]\right)+\pi\left(d_{n}^{1},\left[d_{k}^{1}, d_{m}^{1}\right]\right)+\pi\left(d_{k}^{1},\left[d_{m}^{1}, d_{n}^{1}\right]\right)=0,
$$

a similar calculation as above shows that

$$
(k+n) \pi\left(d_{n}^{1}, d_{k}^{1}\right)=(k-n) \pi\left(d_{0}^{1}, d_{n+k}^{1}\right)
$$

for all $n, k \in \mathbb{Z}$, and

(**) $\quad \frac{2\left(\pi\left(d_{2 m}^{1}, d_{-2 m}^{1}\right)-2 \pi\left(d_{m}^{1}, d_{-m}^{1}\right)\right)}{m^{3}}=\frac{12 \pi\left(d_{m}^{1}, d_{-m}^{1}\right)}{m\left(m^{2}-n^{2}\right)}+\frac{12 \pi\left(d_{n}^{1}, d_{-n}^{1}\right)}{n\left(n^{2}-m^{2}\right)}$

for all $m, n \neq 0, m^{2} \neq n^{2}$. Thus

$$
\phi_{1}:=\frac{2\left(\pi\left(d_{2 m}^{1}, d_{-2 m}^{1}\right)-2 \pi\left(d_{m}^{1}, d_{-m}^{1}\right)\right)}{m^{3}}
$$

is independent of $m, m \neq 0$.

Now we define

$$
\begin{aligned}
& \tilde{d}_{m}^{1}:=d_{m}^{1}+\frac{1}{m} \pi\left(d_{0}^{1}, d_{m}^{1}\right) \quad \text { where } m \neq 0, \\
& \tilde{d}_{0}^{1}:=d_{0}^{1}-\frac{1}{2} \pi\left(d_{1}^{1}, d_{-1}^{1}\right), \\
& \tilde{d}_{m}^{0}:=d_{m}^{0}+\frac{1}{m} \pi\left(d_{0}^{1}, d_{m}^{0}\right) \text { where } m \neq 0, \\
& \tilde{d}_{0}^{0}:=d_{0}^{0}-\frac{1}{2} \pi\left(d_{1}^{0}, d_{-1}^{0}\right),
\end{aligned}
$$

and

$$
\tilde{\mathfrak{g}}\{0,1\}:=\sum_{m \in \mathbb{Z}} \mathbb{C} \tilde{d}_{m}^{1}+\sum_{m \in \mathbb{Z}} \mathbb{C} \tilde{d}_{m}^{0}+\mathbb{C} \phi_{1}+\mathbb{C} \phi_{2}+\mathbb{C} \phi_{3}
$$


Proposition 2. $\tilde{\mathfrak{g}}\{0,1\}$ is the universal central extension of $\mathfrak{g}\{0,1\}$ and the Lie brackets of $\tilde{\mathfrak{g}}\{0,1\}$ are

$$
\begin{aligned}
& {\left[\tilde{d}_{m}^{1}, \tilde{d}_{n}^{1}\right]=(n-m) \tilde{d}_{m+n}^{1}+\frac{m^{3}-m}{12} \delta_{m+n, 0} \phi_{1},} \\
& {\left[\tilde{d}_{m}^{1}, \tilde{d}_{n}^{0}\right]=n \tilde{d}_{m+n}^{0}+\frac{m(m-1)}{2} \delta_{m+n, 0} \phi_{2},} \\
& {\left[\tilde{d}_{m}^{0}, \tilde{d}_{n}^{0}\right]=m \delta_{m+n, 0} \phi_{3},}
\end{aligned}
$$

where $\phi_{1}, \phi_{2}, \phi_{3}$ are in the centre.

Proof. Clearly,

$$
\tilde{\mathfrak{g}}\{0,1\} \subseteq \mathfrak{g}\{0,1\} \oplus \frac{\pi \mathfrak{g}\{0,1\}}{I},
$$

and we have

$$
\begin{aligned}
& {\left[\tilde{d}_{m}^{1}, \tilde{d}_{n}^{1}\right] } \\
&= {\left[d_{m}^{1}, d_{n}^{1}\right]+\pi\left(d_{m}^{1}, d_{n}^{1}\right) } \\
&=(n-m) d_{m+n}^{1}+\frac{n-m}{n+m} \pi\left(d_{0}^{1}, d_{m+n}^{1}\right) \quad(u \operatorname{sing}(*)) \\
&=(n-m)\left(\tilde{d}_{m+n}^{1}-\frac{1}{m+n} \pi\left(d_{0}^{1}, d_{m}^{1}\right)\right)+\frac{n-m}{n+m} \pi\left(d_{0}^{1}, d_{m+n}^{1}\right) \\
&=(n-m) \pi d_{m+n}^{1}, \quad(\text { provided that } m+n \neq 0), \\
& {\left[\tilde{d}_{m}^{1}, \tilde{d}_{-m}^{1}\right] } \\
&=-2 m d_{0}^{1}+\pi\left(d_{m}^{1}, d_{-m}^{1}\right) \\
&=-2 m\left(\tilde{d}_{0}^{1}+\frac{1}{2} \pi\left(d_{1}^{1}, d_{-1}^{1}\right)\right)+\pi\left(d_{m}^{1}, d_{-m}^{1}\right) \\
&=-2 m \tilde{d}_{0}^{1}-m \pi\left(d_{1}^{1}, d_{-1}^{1}\right)+m\left(m^{2}-1\right) \frac{1}{12} \phi_{1}+m \pi\left(d_{1}^{1}, d_{-1}^{1}\right) \\
&(u \operatorname{sing}(* *) \text { with } n=1) \\
&=-2 m \tilde{d}_{0}^{1}+\frac{m^{3}-m}{12} \delta_{m+n, 0} \phi_{1} .
\end{aligned}
$$

We can verify the other two commutators similarly. So $\tilde{\mathbf{g}}\{0,1\}$ is a Lie subalgebra of $\mathfrak{g}\{0,1\} \oplus\left(\Lambda^{2} \mathfrak{g}\{0,1\} / I\right)$. Moreover,

$$
\begin{aligned}
& {\left[\mathfrak{g}\{0,1\} \oplus \frac{\Lambda^{2} \mathfrak{g}\{0,1\}}{I}, \mathfrak{g}\{0,1\} \oplus \frac{\Lambda^{2} \mathfrak{g}\{0,1\}}{I}\right]} \\
& \quad=[\tilde{\mathfrak{g}}\{0,1\}, \tilde{\mathfrak{g}}\{0,1\}] \\
& \quad=\tilde{\mathfrak{g}}\{0,1\},
\end{aligned}
$$

so $\tilde{\mathfrak{g}}\{0,1\}$ is the universal central extension of $\mathfrak{g}\{0,1\}$. 


\section{Admissible Modules}

Let $\mathfrak{h} \subseteq \mathfrak{g}$ be a homogeneous Lie subalgebra and $\mathfrak{h}=\sum_{k \in \mathbb{Z}} \mathfrak{h}_{k}$. Then $\mathfrak{h}_{0}$ is an abelian Lie subalgebra of $\mathfrak{h}$. A $\mathfrak{h}$-module $V$ is called admissible if

(i) $V=\sum_{\lambda \in \mathfrak{h}_{0}^{*}} V_{\lambda}$, where $\mathfrak{h}_{0}^{*}$ is the dual space of $\mathfrak{h}_{0}$, and

$$
V_{\lambda}=\left\{v \in V \mid X \cdot v=\lambda(X) v \quad \forall X \in \mathfrak{h}_{0}\right\} .
$$

(ii) $\operatorname{dim}\left(V_{\lambda}\right)<\infty$ for all $\lambda \in \mathfrak{h}_{0}^{*}$.

For $g\{x\}$, I. Kaplansky [Kap] and I. Kaplansky and L.J. Santharoubane [KS] proved the following result:

If $V=\sum_{k \in \mathbb{Z}} \mathbb{C} v_{k}$ is a $\mathfrak{g}\{x\}$-module such that

$$
\begin{gathered}
d_{m}^{1} \cdot v_{k} \in \mathbb{C} v_{m+k} \quad \forall m, k \in \mathbb{Z}, \\
d_{1}^{1} \cdot v_{k} \neq 0, \quad d_{-1}^{1} \cdot v_{k} \neq 0 \quad \forall k \in \mathbb{Z},
\end{gathered}
$$

then there exists $\alpha, \beta \in \mathbb{C}$ such that

$$
d_{m}^{1} \cdot v_{k}=(k+\alpha m+\beta) v_{m+k} \quad \forall m, k \in \mathbb{Z} .
$$

In this section we prove similar results for $\mathfrak{g}$ and $\mathfrak{g}\{0,1\}$.

Proposition 1. Let $V=\sum_{k \in \mathbb{Z}} \mathbb{C} v_{k}$ be a $\mathfrak{g}\{0,1\}$-module such that

$$
d_{m}^{1} \cdot v_{k} \in \mathbb{C} v_{m+k}, \quad d_{m}^{0} \cdot v_{k} \in \mathbb{C} v_{m+k},
$$

and

$$
d_{1}^{1} \cdot v_{k} \neq 0, \quad d_{-1}^{1} \cdot v_{k} \neq 0
$$

for all $k \in \mathbb{Z}$. Then there exists $\alpha, \beta, \gamma \in \mathbb{C}$ such that

(i) $d_{m}^{1} \cdot v_{k}=(k+\alpha m+\beta) v_{m+k}$.

(ii) If $\alpha \neq 0,1$, then $d_{m}^{0} \cdot v_{k}=\gamma v_{m+k}$ for all $m, k \in \mathbb{Z}$.

If $\alpha=0$, then

$$
d_{m}^{0} \cdot v_{k}=\gamma v_{m+k} \quad \forall m, k \in \mathbb{Z}
$$

or

$$
d_{m}^{0} \cdot v_{k}=\frac{(k+\beta) \gamma}{k+m+\beta} v_{m+k} \quad \forall m, k \in \mathbb{Z}
$$

If $\alpha=1$, then

$$
d_{m}^{0} \cdot v_{k}=\gamma v_{m+k} \quad \forall m, k \in \mathbb{Z}
$$

or

$$
d_{m}^{0} \cdot v_{k}=\frac{(k+m+\beta) \gamma}{k+\beta} v_{m+k} \quad \forall m, k \in \mathbb{Z} .
$$

Proof. (i) follows from the result of Kaplansky and Santharoubane's quoted above.

(ii) First we note by assumption,

$$
d_{1}^{1} v_{k}=(k+\alpha+\beta) v_{k+1} \neq 0, \quad d_{-1}^{1} v_{k}=(k-\alpha+\beta) v_{k-1} \neq 0,
$$


for all $k \in \mathbb{Z}$. So $\beta \pm \alpha \notin \mathbb{Z}$. Assume that $d_{m}^{0} \cdot v_{k}=f(m, k) v_{m+k}$. Then from

$$
d_{m}^{0} d_{n}^{0} v_{k}=d_{n}^{0} d_{m}^{0} v_{k}
$$

and

$$
\left[d_{m}^{1}, d_{n}^{0}\right] v_{k}=\left(d_{m}^{1} d_{n}^{0}-d_{n}^{0} d_{m}^{1}\right) v_{k}
$$

we obtain

$$
\left\{\begin{array}{l}
f(n, k) f(m, n+k)=f(m, k) f(n, m+k) \\
n f(m+n, k)=f(n, k)(n+k+\alpha m+\beta)-f(n, m+k)(k+\alpha m+\beta)
\end{array}\right.
$$

for all $m, n, k \in \mathbb{Z}$.

Let $k=0$ in (1). Then

$$
\left\{\begin{array}{l}
f(n, 0) f(m, n)=f(m, 0) f(n, m) \\
n f(m+n, 0)=f(n, 0)(n+\alpha m+\beta)-f(n, m)(\alpha m+\beta),
\end{array}\right.
$$

or

$$
\left\{\begin{array}{l}
f(n, 0) f(m, n)=f(m, 0) f(n, m) \\
f(n, m)(\alpha m+\beta)=f(n, 0)(n+\alpha m+\beta)-n f(m+n, 0) .
\end{array}\right.
$$

Multiplying both sides of the second identity of (1) by $(\alpha k+\beta)(\alpha(m+k)+\beta)$ and using the second identity of (3), we obtain

$$
\begin{aligned}
& n(\alpha(m+k)+\beta)(f(m+n, 0)(m+n+\alpha k+\beta)-(m+n) f(m+n+k, 0)) \\
& =(\alpha(m+k)+\beta)(n+k+\alpha m+\beta)(f(n, 0)(n+k \alpha+\beta)-n f(n+k, 0)) \\
& \quad-(\alpha k+\beta)(k+\alpha m+\beta)(f(n, 0)(n+\alpha(m+k)+\beta)-n f(m+n+k, 0)) .
\end{aligned}
$$

Let $m=-1, n=1$ in (4). Then

$$
\begin{aligned}
(\alpha(k-1) & +\beta)(k+1-\alpha+\beta) f(k+1,0) \\
= & (\alpha k+\beta)(k-\alpha+\beta) f(k, 0) \\
& +((\alpha(k-1)+\beta)(\alpha k+\beta)+(1-\alpha)(\beta-\alpha)) f(1,0) \\
& \quad-(\alpha(k-1)+\beta)(\alpha k+\beta) f(0,0) .
\end{aligned}
$$

With $k=-1$ in (5), we get

$$
(\beta-\alpha-1) f(-1,0)-2(\beta-2 \alpha) f(0,0)+(\beta-3 \alpha+1) f(1,0)=0 .
$$

Let $m=k=1, n=-1$ in (4). Then

$$
(\beta+3 \alpha-1) f(-1,0)-2(\beta+2 \alpha) f(0,0)+(\beta+\alpha+1) f(1,0)=0 .
$$

Combining (6) and (7) we get

$$
\alpha(1-\alpha) f(0,0)=\alpha(1-\alpha) f(1,0) .
$$

We consider the following three different cases:

(a). $\alpha \neq 0,1$. 
In this case, $f(0,0)=f(1,0)$ and (5) becomes

$$
\begin{aligned}
& (\alpha(k-1)+\beta)(k+1-\alpha+\beta) f(k+1,0) \\
& \quad=(\alpha k+\beta)(k-\alpha+\beta) f(k, 0)+(1-\alpha)(\beta-\alpha) f(0,0) .
\end{aligned}
$$

If $0 \notin \alpha \mathbb{Z}+\beta$, then by induction on $k$ and the fact $\beta \pm \alpha \notin \mathbb{Z}$, we get $f(k, 0)=f(0,0)$ for all $k \in \mathbb{Z}$. Then by (3), we get

$$
f(m, n)=f(0,0) \quad \forall m, n \in \mathbb{Z} .
$$

Now suppose $\alpha l+\beta=0$, for some $l \in \mathbb{Z}$. Since $\beta \pm \alpha \notin \mathbb{Z}, l=0$, or $l \geqq 2$, or $l \leqq-2$.

If $l=0$, then $\beta=0$ and (8) becomes

$$
(k-1)(k+1-\alpha) f(k+1,0)=k(k-\alpha) f(k, 0)-(1-\alpha) f(0,0) .
$$

With $k=-1$ in (9), we get

$$
2 \alpha f(0,0)=(1+\alpha) f(-1,0)-(1-\alpha) f(0,0) .
$$

Hence

$$
f(-1,0)=f(0,0)
$$

By induction on $k$ we have

$$
f(k, 0)=f(0,0)
$$

for all $k \leqq 1$. With $k=2$ in (9) we get

$$
(3-\alpha) f(3,0)=2(2-\alpha) f(2,0)-(1-\alpha) f(0,0) .
$$

By induction on $k$ we have

$$
(k-\alpha) f(k, 0)=(k-1)(2-\alpha) f(2,0)-(k-2)(1-\alpha) f(0,0)
$$

for all $k \geqq 2$. With $n=-1, m=2$ in the second identity of (3), we obtain

$$
\begin{aligned}
2 \alpha f(-1,2) & =(2 \alpha-1) f(-1,0)+f(1,0) \\
& =2 \alpha f(0,0)
\end{aligned}
$$

or

$$
f(-1,2)=f(0,0) \text {. }
$$

With $m=-1, n=2$ in the second identity of (3), we obtain

$$
-\alpha f(2,-1)=(-\alpha+2) f(2,0)-2 f(1,0) .
$$

With these and by the first identity of (3), we get

$$
-\alpha f(2,0) f(0,0)=(2-\alpha) f(2,0) f(0,0)-2 f(0,0) f(0,0),
$$

or

$$
f(0,0)(f(2,0)-f(0,0))=0 .
$$

If $f(0,0) \neq 0$, then $f(2,0)=f(0,0)$. Then by $(10), f(k, 0)=f(0,0)$ for all $k \in \mathbb{Z}$.

If $f(0,0)=0$, then $(10)$ becomes

$$
(k-\alpha) f(k, 0)=(k-1)(2-\alpha) f(2,0)
$$


for all $k \geqq 2$. Using this and letting $n=1, m=2$ in the second identity of (3), we get

$$
2 \alpha f(1,2)=-f(3,0) \text {. }
$$

With this and the fact $f(1,0)=0$, and with $m=1, n=2$ in the first identity of (3), we get $f(2,0) f(3,0)=0$. Hence by $(11), f(2,0)=f(3,0)=0$. These imply $f(k, 0)=$ $0=f(0,0)$ for all $k \in \mathbb{Z}$. Now by the second identity of (3),

$$
f(n, m)=f(0,0)
$$

for all $m, n \in \mathbb{Z}$.

If $l \geqq 2$, then

$$
\alpha k+\beta \neq 0
$$

for all $k<l$, and by (8),

$$
f(l, 0)=\cdots=f(1,0)=f(0,0)=\cdots .
$$

From this and with $m=l$ and $n=1$ in the second identity of (3), we obtain $f(l+1,0)=f(l, 0)$. Then by $(8)$,

$$
f(l, 0)=f(l+1,0)=\cdots .
$$

So $f(k, 0)=f(0,0) \forall k \in \mathbb{Z}$. Now by the second identity of (3) we obtain $f(n, m)=$ $f(0,0)$ for all $m \neq l$.

If $f(0,0) \neq 0$, multiplying both sides of the first identity of (3) by $f(0,0)^{-1}=$ $f(n, 0)^{-1}=f(m, 0)^{-1}$, we get $f(m, n)=f(n, m) \forall m, n \in \mathbb{Z}$. Hence

$$
f(m, n)=f(0,0) \quad \forall m, n \in \mathbb{Z} .
$$

If $f(0,0)=0$, then $f(n, m)=0$ for all $m \neq l$. From this and with $m \neq 0, k=l$ in the second identity of (1), we get

$$
n f(m+n, l)=f(n, l)(n+l+\alpha m+\beta) .
$$

With $n=0$ and $l+\alpha m+\beta \neq 0$ in (12), we obtain $f(0, l)=0$. With $m+n=0$ in (12), we obtain $f(n, l)=0$ for all $n \gg 0$. Now (12) gives us $n f(m+n, l)=0$ for all $n \gg 0$ and all $m \in \mathbb{Z}$. So $f(n, l)=0=f(0,0)$ for all $n \in \mathbb{Z}$. We have proved that

$$
f(m, n)=0=f(0,0) \quad \forall m \in \mathbb{Z} .
$$

The case of $l \leqq-2$ can be proved similarly. So for $\alpha \neq 0,1$, we have

$$
f(m, n)=f(0,0) \quad \forall m, n \in \mathbb{Z} .
$$

(b). $\alpha=0$.

In this case $\beta \notin \mathbb{Z}$, and (3) becomes

$$
\left\{\begin{array}{l}
f(n, 0) f(m, n)=f(m, 0) f(n, m) \\
\beta f(n, m)=(n+\beta) f(n, 0)-n f(m+n, 0),
\end{array}\right.
$$

and (5) becomes

$$
(k+1+\beta) f(k+1,0)=(k+\beta) f(k, 0)+(1+\beta) f(1,0)-\beta f(0,0) .
$$


By induction on $k$, we obtain from (14) that

$$
f(k, 0)=\frac{k(1+\beta)}{k+\beta} f(1,0)-\frac{(k-1) \beta}{k+\beta} f(0,0) .
$$

Thus

$$
f(-1,0)=-\frac{1+\beta}{\beta-1} f(1,0)+\frac{2 \beta}{\beta-1} f(0,0) .
$$

With $n=1, m=-1$ in (13), we get

$$
f(1,0) f(-1,1)=f(-1,0) f(1,-1)
$$

and

$$
f(1,-1)=\frac{1+\beta}{\beta} f(1,0)-\frac{1}{\beta} f(0,0) .
$$

With $n=-1, m=1$ in the second identity of (13), we get

$$
f(-1,1)=-\frac{1+\beta}{\beta} f(1,0)+\frac{1+2 \beta}{\beta} f(0,0) .
$$

Combining above results, we get

$$
(1+\beta) f^{2}(1,0)-(1+2 \beta) f(1,0) f(0,0)+\beta f^{2}(0,0)=0,
$$

or

$$
(f(1,0)-f(0,0))((1+\beta) f(1,0)-\beta f(0,0))=0 .
$$

Hence

$$
f(1,0)=f(0,0)
$$

or

$$
f(1,0)=\frac{\beta}{1+\beta} f(0,0) .
$$

If $f(1,0)=f(0,0)$, by $(15), f(k, 0)=f(0,0) \forall k \in \mathbb{Z}$. Then by (13),

$$
f(n, m)=f(0,0) \quad \forall m, n \in \mathbb{Z} .
$$

If $f(1,0)=\frac{\beta}{1+\beta} f(0,0)$, by $(15), f(k, 0)=\frac{\beta}{k+\beta} f(0,0) \forall k \in \mathbb{Z}$. Then by (13), we obtain

$$
f(n, m)=\frac{m+\beta}{n+m+\beta} f(0,0) \quad \forall m, n \in \mathbb{Z} .
$$

(c). $\alpha=1$.

In this case $\beta \notin \mathbb{Z}$ and (3) becomes

$$
\left\{\begin{array}{l}
f(n, 0) f(m, n)=f(m, 0) f(n, m) \\
f(n, m)(m+\beta)=f(n, 0)(n+m+\beta)-n f(m+n, 0),
\end{array}\right.
$$

and (5) becomes

$$
f(k+1,0)=f(k, 0)+f(1,0)-f(0,0) .
$$


With $k=-1$ in (17), we get

$$
f(-1,0)=2 f(0,0)-f(1,0) .
$$

With $n=-1, m=1$ in (16), we get

$$
f(-1,0) f(1,-1)=f(1,0) f(-1,1),
$$

and

$$
f(-1,1)=\frac{\beta}{1+\beta} f(-1,0)+\frac{1}{1+\beta} f(0,0) .
$$

Again, with $n=1, m=-1$ in the second identity of (16), we get

$$
f(1,-1)=\frac{\beta}{\beta-1} f(1,0)-\frac{1}{\beta-1} f(0,0) .
$$

Combining above results, we have

$$
(\beta-1) f^{2}(1,0)-2 \beta f(1,0) f(-1,0)+(1+\beta) f^{2}(-1,0)=0,
$$

or

$$
((\beta-1) f(1,0)-(\beta+1) f(-1,0))(f(1,0)-f(-1,0))=0
$$

Hence

$$
f(-1,0)=f(1,0)
$$

or

$$
f(-1,0)=\frac{\beta-1}{\beta+1} f(1,0)
$$

Then from (18),

$$
f(1,0)=f(0,0)
$$

or

$$
f(1,0)=\frac{1+\beta}{\beta} f(0,0) .
$$

If $f(1,0)=f(0,0)$, by $(17), f(k, 0)=f(0,0) \forall k \in \mathbb{Z}$. Then by the second identity of (16),

$$
f(n, m)=f(0,0) \quad \forall m, n \in \mathbb{Z} .
$$
of (16),

If $f(1,0)=\frac{1+\beta}{\beta} f(0,0)$, by $(17), f(k, 0)=\frac{k+\beta}{\beta} f(0,0)$. Then by the second identity

$$
f(n, m)=\frac{m+n+\beta}{m+\beta} f(0,0) \quad \forall m, n \in \mathbb{Z} .
$$

Remark. It is easy to check that the action of $\mathfrak{g}\{0,1\}$ on $V$ given by (i), (ii) in Proposition 1 indeed make $V$ into a $\mathfrak{g}\{0,1\}$-module. Moreover, if $\gamma \neq 0$, then $V$ is an irreducible $g\{0,1\}$-module. 
Now we suppose that $V=\sum_{k \in \mathbb{Z}} \mathbb{C} v_{k}$ is a g-module and satisfies the following conditions:

$d_{m}^{r} \cdot v_{k} \in \mathbb{C} v_{m+k}$ for all $m, k \in \mathbb{Z}, r \in \mathbb{Z} \geqq 0$, and $d_{1}^{1} \cdot v_{k} \neq 0, d_{-1}^{1} \cdot v_{k} \neq 0$ for all $k \in \mathbb{Z}$.

Since $V$ is a $\mathfrak{g}\{0,1\}$-module by restriction, by Proposition 1 , there exists $\alpha, \beta, \gamma \in \mathbb{C}$ such that one of the following occurs:

$$
d_{m}^{1} \cdot v_{k}=(k+\alpha m+\beta) v_{m+k}, \quad d_{m}^{0} \cdot v_{k}=\gamma v_{m+k},
$$

where $\beta \pm \alpha \notin \mathbb{Z}$.

$$
d_{m}^{1} \cdot v_{k}=(k+\beta) v_{m+k}, \quad d_{m}^{0} \cdot v_{k}=\frac{(k+\beta) \gamma}{m+k+\beta} v_{m+k},
$$

where $\alpha=0, \beta \notin \mathbb{Z}$.

$$
d_{m}^{1} \cdot v_{k}=(k+m+\beta) v_{m+k}, \quad d_{m}^{0} \cdot v_{k}=\frac{(k+m+\beta) \gamma}{k+\beta} v_{m+k},
$$

where $\alpha=1, \beta \notin \mathbb{Z}$.

Claim 1. $\gamma \neq 0$.

Proof. If $\gamma=0$, then $d_{m}^{0} v_{k}=0 \forall m, k \in \mathbb{Z}$. Comparing

$$
\left[d_{m}^{2}, d_{n}^{0}\right] \cdot v_{k}=2 n d_{m+n}^{1} \cdot v_{k}=2 n(k+\alpha(m+n)+\beta) v_{m+n+k}
$$

with

$$
\left(d_{m}^{2} d_{n}^{0}-d_{n}^{0} d_{m}^{2}\right) \cdot v_{k}=-d_{n}^{0} d_{m}^{2} \cdot v_{k}=0
$$

we get

$$
2 n(k+\alpha(m+n)+\beta) \cdot v_{m+n+k}=0 \quad \forall m, n, k \in \mathbb{Z} .
$$

This is a contradiction.

Assume that $d_{m}^{r} v_{k}=f_{r}(m, k) v_{m+k}, \forall m, k \in \mathbb{Z}, \forall r \in \mathbb{Z}_{\geqq 0}$. Since

$$
\begin{aligned}
{\left[d_{m}^{r}, d_{0}^{2}\right] } & =-2 m d_{m}^{r+1}-m^{2} d_{m}^{r}, \\
{\left[d_{m}^{r}, d_{0}^{2}\right] v_{k} } & =\left(-2 m f_{r+1}(m, k)-m^{2} f_{r}(m, k)\right) v_{m+k} .
\end{aligned}
$$

On the other hand

$$
\left(d_{m}^{r} d_{0}^{2}-d_{0}^{2} d_{m}^{r}\right) v_{k}=\left(f_{2}(0, k) f_{r}(m, k)-f_{r}(m, k) f_{2}(0, m+k)\right) v_{m+k} .
$$

So we have

$$
-2 m f_{r+1}(m, k)=f_{r}(m, k)\left(m^{2}+f_{2}(0, k)-f_{2}(0, m+k)\right) .
$$

Since

$$
\left[d_{m}^{r}, d_{n}^{0}\right]=\left(\begin{array}{c}
r \\
1
\end{array}\right) n d_{m+n}^{r-1}+\left(\begin{array}{c}
r \\
2
\end{array}\right) n^{2} d_{m+n}^{r-2}+\cdots+\left(\begin{array}{c}
r \\
r
\end{array}\right) n^{r} d_{m+n}^{0}
$$

$\left[d_{m}^{r}, d_{n}^{0}\right] v_{k}$

$$
=\left(\left(\begin{array}{l}
r \\
1
\end{array}\right) n f_{r-1}(m+n, k)+\left(\begin{array}{c}
r \\
2
\end{array}\right) n^{2} f_{r-2}(m+n, k)+\cdots+\left(\begin{array}{c}
r \\
r
\end{array}\right) n^{r} f_{0}(m+n, k)\right) v_{m+n+k} .
$$

On the other hand,

$$
\left(d_{m}^{r} d_{n}^{0}-d_{n}^{0} d_{m}^{r}\right) v_{k}=\left(f_{0}(n, k) f_{r}(m, n+k)-f_{r}(m, k) f_{0}(n, m+k)\right) v_{m+n+k} .
$$


So we have

$$
\begin{gathered}
\left(\begin{array}{c}
r \\
1
\end{array}\right) n f_{r-1}(m+n, k)+\left(\begin{array}{c}
r \\
2
\end{array}\right) n^{2} f_{r-2}(m+n, k)+\cdots+\left(\begin{array}{c}
r \\
r
\end{array}\right) n^{r} f_{0}(m+n, k) \\
=f_{0}(n, k) f_{r}(m, n+k)-f_{r}(m, k) f_{0}(n, m+k) .
\end{gathered}
$$

In particular, if $r=2$ and $m=0$, we get

$$
f_{0}(n, k)\left(f_{2}(0, n+k)-f_{2}(0, k)\right)=2 n f_{1}(n, k)+n^{2} f_{0}(n, k) .
$$

By Claim $1, f_{0}(n, k) \neq 0$. So

$$
f_{2}(0, n+k)-f_{2}(0, k)=2 n \frac{f_{1}(n, k)}{f_{0}(n, k)}+n^{2} \quad \forall n, k \in \mathbb{Z} .
$$

Then from (19),

$$
f_{r+1}(m, k)=f_{r}(m, k) \frac{f_{1}(m, k)}{f_{0}(m, k)} \quad \text { if } m \neq 0 .
$$

(a) $f_{0}(n, k)=\gamma, \quad f_{1}(n, k)=k+\alpha n+\beta$.

Claim 2. $\gamma= \pm 1$.

If $\gamma=1$, then $f_{r}(m, k)=(k+\beta)^{r}$ for all $m, k \in \mathbb{Z}, r \in \mathbb{Z} \geqq 0$.

If $\gamma=-1$, then $f_{r}(m, k)=(-1)^{r-1}(k+m+\beta)^{r}$ for all $m, k \in \mathbb{Z}, r \in \mathbb{Z} \geqq 0$.

Proof. In this case, (21) becomes

$$
f_{2}(0, n+k)-f_{2}(0, k)=\frac{2 n(k+\alpha n+\beta)}{\gamma}+n^{2} .
$$

With $k=0$ in (23), we get

$$
f_{2}(0, n)-f_{2}(0,0)=\frac{2 n(\alpha n+\beta)}{\gamma}+n^{2} .
$$

With $n+k=0$ in (23) and replacing $n$ by $-n$, we get

$$
f_{2}(0, n)-f_{2}(0,0)=\frac{2 n(n-\alpha n+\beta)}{\gamma}-n^{2} .
$$

These imply that $\gamma=1-2 \alpha$.

Now (22) becomes

$$
f_{r+1}(m, k)=f_{r}(m, k) \frac{k+\alpha m+\beta}{1-2 \alpha} \text { if } m \neq 0 .
$$

By induction on $r$, we get

$$
f_{r}(m, k)=(1-2 \alpha)\left(\frac{k+\alpha m+\beta}{1-2 \alpha}\right)^{r} \text { if } m \neq 0 \text {. }
$$

With $m+n=0$ in (20), we have

$$
\begin{aligned}
\left(\begin{array}{c}
r \\
1
\end{array}\right) & n f_{r-1}(0, k)+\left(\begin{array}{c}
r \\
2
\end{array}\right) n^{2} f_{r-2}(0, k)+\cdots+\left(\begin{array}{c}
r \\
r
\end{array}\right) n^{r} f_{0}(0, k) \\
& =(1-2 \alpha)\left(f_{r}(-n, n+k)-f_{r}(-n, k)\right) \\
& =(1-2 \alpha)^{2-r}\left((k+\beta+(1-\alpha) n)^{r}-(k+\beta-\alpha n)^{r}\right) .
\end{aligned}
$$


In particular, if $r=3$ and $k=0$, then

$$
\begin{aligned}
& 3 n f_{2}(0,0)+3 n^{2} f_{1}(0,0)+n^{3} f_{0}(0,0) \\
& \quad=\frac{1}{1-2 \alpha}\left(3 \beta^{2} n+3 \beta(1-2 \alpha) n^{2}+\left(1-3 \alpha+3 \alpha^{2}\right) n^{3}\right) .
\end{aligned}
$$

Since $n$ is arbitrary,

$$
1-2 \alpha=f_{0}(0,0)=\frac{1}{1-2 \alpha}\left(1-3 \alpha+3 \alpha^{2}\right) .
$$

So $\alpha=0$ or 1 , and $\gamma=1-2 \alpha=1$ or -1 .

If $\gamma=1$, then $\alpha=0$. With $n=1$ in (24), we have

$$
\begin{gathered}
\left(\begin{array}{l}
r \\
1
\end{array}\right) f_{r-1}(0, k)+\left(\begin{array}{c}
r \\
2
\end{array}\right) f_{r-1}(0, k)+\cdots+\left(\begin{array}{c}
r \\
r
\end{array}\right) f_{0}(0, k) \\
=\left(\begin{array}{c}
r \\
1
\end{array}\right)(k+\beta)^{r-1}+\left(\begin{array}{c}
r \\
2
\end{array}\right)(k+\beta)^{r-2}+\cdots+\left(\begin{array}{c}
r \\
r
\end{array}\right) .
\end{gathered}
$$

By induction on $r$, we get

$$
f_{r}(0, k)=(k+\beta)^{r} \text {. }
$$

Hence

$$
f_{r}(m, k)=(k+\beta)^{r} \quad \forall m, k \in \mathbb{Z}, \quad r \in \mathbb{Z} \geqq 0 .
$$

If $\gamma=-1$, then $\alpha=1$. With $n=1$ in (24), we have

$$
\begin{aligned}
& \left(\begin{array}{l}
r \\
1
\end{array}\right) f_{r-1}(0, k)+\left(\begin{array}{l}
r \\
2
\end{array}\right) f_{r-1}(0, k)+\cdots+\left(\begin{array}{c}
r \\
r
\end{array}\right) f_{0}(0, k) \\
& =(-1)^{2-r}\left((k+\beta)^{r}-(k+\beta-1)^{r}\right) \\
& =\left(\begin{array}{l}
r \\
1
\end{array}\right)(-1)^{r}(k+\beta)^{r-1}+\left(\begin{array}{c}
r \\
2
\end{array}\right)(-1)^{r-1}(k+\beta)^{r-2}+\cdots+\left(\begin{array}{l}
r \\
r
\end{array}\right)(-1) .
\end{aligned}
$$

By induction on $r$, we get

$$
f_{r}(0, k)=(-1)^{r+1}(k+\beta)^{r} .
$$

Hence,

$$
f_{r}(m, k)=(-1)^{r-1}(k+m+\beta)^{r} \quad \forall m, k \in \mathbb{Z}, \quad r \in \mathbb{Z}_{\geqq 0} .
$$

We complete the proof of Claim 2.

(b) $f_{0}(m, k)=\frac{(k+\beta) \gamma}{m+k+\beta}, \quad f_{1}(m, k)=k+\beta$.

Claim 3. $f_{r}(m, k)=(-1)^{r-1}(k+m+\beta)^{r-1}(k+\beta)$.

Proof. In this case, (21) becomes

$$
f_{2}(0, n+k)-f_{2}(0, k)=2 n \frac{n+k+\beta}{\gamma}+n^{2} .
$$

With $k=0$ in (25), we get

$$
f_{2}(0, n)-f_{2}(0,0)=\frac{2 n(n+\beta)}{\gamma}+n^{2} .
$$


With $n+k=0$ in (25) and replacing $n$ by $-n$, we get

$$
f_{2}(0, n)-f_{2}(0,0)=\frac{2 n \beta}{\gamma}-n^{2} .
$$

These imply that $\gamma=-1$. Hence (22) becomes

$$
f_{r+1}(m, k)=-(m+k+\beta) f_{r}(m, k) \text { where } m \neq 0 .
$$

By induction on $r$, we get

$$
f_{r}(m, k)=(-1)^{r-1}(k+m+\beta)^{r-1}(k+\beta)
$$

for all $k, m \in \mathbb{Z}, m \neq 0$, and all $r \in \mathbb{Z}_{\geqq 0}$. Using this and with $m=-1, n=1$ in (20), we get

$$
\begin{aligned}
& \left(\begin{array}{c}
r \\
1
\end{array}\right) f_{r-1}(0, k)+\left(\begin{array}{c}
r \\
2
\end{array}\right) f_{r-2}(0, k)+\cdots+\left(\begin{array}{c}
r \\
1
\end{array}\right) f_{0}(0, k) \\
& \quad=f_{0}(1, k) f_{r}(-1, k+1)-f_{r}(-1, k) f_{0}(1, k-1) \\
& \quad=(-1)^{r}\left((k+\beta)^{r}-(k+\beta-1)^{r}\right) \\
& \quad=-\left(\left(\begin{array}{c}
r \\
1
\end{array}\right)(-1)^{r-1}(k+\beta)^{r-1}+\left(\begin{array}{c}
r \\
2
\end{array}\right)(-1)^{r-2}(k+\beta)^{r-2}+\cdots+\left(\begin{array}{c}
r \\
r
\end{array}\right)\right) .
\end{aligned}
$$

By induction on $r$, we obtain

$$
f_{r}(0, k)=(-1)^{r-1}(k+\beta)^{r} .
$$

Hence we complete the proof of the Claim 3.

(c) $f_{0}(m, k)=\frac{(m+k+\beta) \gamma}{k+\beta}, \quad f_{1}(m, k)=m+k+\beta$.

An argument similar to the one in (b) shows us that $\gamma=1$ and gives us the following claim.

Claim 4. $f_{r}(m, k)=(k+m+\beta)(k+\beta)^{r-1}$.

Using the fact that $g$ is generated by $d_{0}^{2}, d_{1}^{0}, d_{-1}^{0}$, it is easy to see that if $f_{r}(m, k)$ is defined as in Claim 2, Claim 3, and Claim 4, $d_{m}^{r} \cdot v_{k}=f_{r}(m, k) v_{m+k}$ indeed gives $V$ a $\mathrm{g}$-module structure. In summary, we proved the following proposition.

Proposition 2. Let $V=\sum_{k \in \mathbb{Z}} \mathbb{C} v_{k}$. Define $d_{m}^{r} v_{k}:=f_{r}(m, k) v_{m+k}$, for all $m, k \in \mathbb{Z}$, $r \in \mathbb{Z} \geqq 0$, where $f_{r}(m, k)$ are given by the following:

(i) $f_{r}(m, k)=(k+\beta)^{r}$,

(ii) $f_{r}(m, k)=(-1)^{r-1}(k+m+\beta)^{r}$,

(iii) $f_{r}(m, k)=(-1)^{r-1}(k+m+\beta)^{r-1}(k+\beta)$,

(iv) $f_{r}(m, k)=(k+\beta)^{r-1}(k+m+\beta)$, where $\beta \notin \mathbb{Z}$.

Then $V$ is a $\mathfrak{g}$-module and $d_{-1}^{1} v_{k} \neq 0, d_{1}^{1} v_{k} \neq 0$. Conversely, if $V=\sum_{k} \mathbb{C} v_{k}$ is a g-module such that $d_{m}^{r} v_{k} \in \mathbb{C} v_{m+k}$ and $d_{-1}^{1} v_{k} \neq 0, d_{1}^{1} v_{k} \neq 0$, then the $\mathfrak{g}$-module structure of $V$ is given by one of (i), (ii), (iii) and (iv). 
Remark. Let $p(x)=\sum_{i} a_{i} x^{i} \in \mathbb{C}[x]$. Then any $\mathfrak{g}$-module becomes, by restriction to $\mathfrak{g}_{\langle p(x)\rangle}$, a $\mathfrak{g}_{\langle p(x)\rangle}$-module. In particular, the $\mathfrak{g}$-module $V$ of Proposition 2 gives rise, by restriction, to a $\mathfrak{g}_{\langle p(x)\rangle}$-module. Precisely, we have $\mathfrak{g}_{\langle p(x)\rangle}$-modules

$$
V_{1, \beta}:=\sum_{k \in \mathbb{Z}} \mathbb{C} v_{k}
$$

with $d_{m}(q(x)) v_{k}=q(k+\beta) v_{m+k}$,

$$
V_{2, \beta}:=\sum_{k \in \mathbb{Z}} \mathbb{C} v_{k}
$$

with $d_{m}(q(x)) v_{k}=-q(-k-m-\beta) v_{m+k}$,

$$
V_{3, \beta}:=\sum_{k \in \mathbb{Z}} \mathbb{C} v_{k}
$$

with $d_{m}(q(x)) v_{k}:=-q(-k-m-\beta) \frac{k+\beta}{m+k+\beta} v_{m+k}$,

and

$$
V_{4, \beta}=\sum_{k \in \mathbb{Z}} \mathbb{C} v_{k}
$$

with $d_{m}(q(x)) v_{k}=q(k+\beta) \frac{m+k+\beta}{k+\beta} v_{m+k}$,

where $q(x) \in\langle p(x)\rangle m, k \in \mathbb{Z}$, and $\beta \notin \mathbb{Z}$.

Proposition 3. Let $\beta \in \mathbb{C} \backslash \mathbb{Z}$. Assume that $k_{1}+\beta, \ldots, k_{\mathbf{r}}+\beta$ are all the distinct roots of $p(x)$ which lie in $\mathbb{Z}+\beta$. Then

$$
U:=\mathbb{C} v_{k_{1}}+\cdots+\mathbb{C} v_{k_{r}}
$$

is the unique maximal proper $\mathfrak{g}_{\langle p(x)\rangle}$-submodule in $V_{i, \beta}, i=1,2,3,4$. And $V_{i, \beta} / U$, $i=1,2,3,4$ are all irreducible.

Proof. We prove only the case of $V_{1, \beta}$. The proof of the cases of $V_{i, \beta} i=2,3,4$ are similar. First note that $\mathfrak{g}_{\langle p(x)\rangle} U=0$, so $U$ is a trivial $\mathfrak{g}_{\langle p(x)\rangle}$-submodule of $V_{1, \beta}$. Now let $K \neq 0$ be a proper $g_{\langle p(x)\rangle}$-submodule in $V_{1, \beta}$ and let $0 \neq X=$ $\sum_{i} a_{i} v_{i} \in K$, where $a_{i} \neq 0, i=i_{1}, \ldots, i_{s}$. If there exists $k$ such that $p\left(i_{k}+\beta\right) \neq 0$, then

$$
0 \neq d_{0}\left(x^{j} p(x)\right) X=\sum_{i} a_{i}(i+\beta)^{j} p(i+\beta) v_{i} \in K
$$

for all $j \in \mathbb{Z}_{\geqq 0}$. Since

$$
\operatorname{det}\left(\begin{array}{cccc}
1 & 1 & \cdots & 1 \\
i_{1}+\beta & i_{2}+\beta & \cdots & i_{s}+\beta \\
\vdots & \vdots & \cdots & \vdots \\
\left(i_{1}+\beta\right)^{s-1} & \left(i_{2}+\beta\right)^{s-1} & \cdots & \left(i_{s}+\beta\right)^{s-1}
\end{array}\right) \neq 0
$$

all $v_{i_{k}} \in K$. Then

$$
d_{m}(p(x)) v_{i_{k}}=p\left(i_{k}+\beta\right) v_{m+i_{k}} \in K \quad \forall m \in \mathbb{Z}
$$


and $K=V_{1, \beta}$. This is a contradiction. So we must have $p(i+\beta)=0$ for $i=i_{1}, \ldots, i_{s}$. Hence $X \in U$.

\section{Highest Weight Modules}

In this section, we discuss the highest weight modules of $\tilde{\mathfrak{g}}$ and $\mathfrak{g}\{0,1\}$. We also define a contravariant forms on Verma modules and give some necessary conditions for these forms to be non-negative.

Recall that we have an imbedding of $\tilde{\mathfrak{g}}$ into $\mathfrak{a}_{\infty}$ by identifying $d_{m}^{r}$ with $\sum_{j} j^{r} E_{j+m, j}$. If we define $L_{m}^{r}=\sum_{i=0}^{r}\left(\begin{array}{c}r \\ i\end{array}\right)\left(\frac{m}{2}\right)^{r-i} d_{m}^{i}$, then $L_{m}^{r}$ is identified as $\sum_{j}\left(j+\frac{m}{2}\right)^{r} E_{j+m, j}$ in $\mathfrak{a}_{\infty}$. Clearly, $\tilde{\mathfrak{g}}=\sum_{m \in \mathbb{Z}, r \in \mathbb{Z}_{\geqq 0}} \mathbb{C} L_{m}^{r}+\mathbb{C} \phi$. By straight calculation, we see that the commutators of $\tilde{g}$ are

$$
\begin{aligned}
{\left[L_{m}^{r}, L_{n}^{s}\right]=} & \sum_{l=0}^{r+s}\left(\sum_{i+k=l}\left(\begin{array}{l}
r \\
i
\end{array}\right)\left(\begin{array}{l}
s \\
k
\end{array}\right)\left(\frac{n}{2}\right)^{i}\left(\frac{m}{2}\right)^{k}\left((-1)^{k}-(-1)^{i}\right)\right) L_{m+n}^{r+s-l} \\
& +\left(\sum_{1 \leqq j \leqq n}\left(j-\frac{n}{2}\right)^{r+s}-\sum_{1 \leqq j \leqq m}\left(j-\frac{m}{2}\right)^{r+s}\right) \delta_{m+n, 0} \phi, \\
{\left[L_{m}^{r}, \phi\right]=} & 0 .
\end{aligned}
$$

Define the anti-linear map

such that

$$
\omega: \mathfrak{a}_{\infty} \rightarrow \mathfrak{a}_{\infty}
$$

$$
\omega\left(\sum_{i} \lambda_{i} E_{i+m, i}\right)=\sum_{i} \bar{\lambda}_{i} E_{i, i+m}, \quad \omega(\phi)=\phi .
$$

It is easy to check that $\omega$ is an anti-involution of $\mathfrak{a}_{\infty}$. Particularly, $\left.\omega\right|_{\tilde{g}}$ is an antiinvolution of $\tilde{\mathfrak{g}}$ and

$$
\omega\left(L_{m}^{r}\right)=\sum_{j}\left(j+\frac{m}{2}\right)^{r} E_{j, j+m}=\sum_{j}\left(j-\frac{m}{2}\right)^{r} E_{j-m, j}=L_{-m}^{r} .
$$

Let

$$
\begin{aligned}
& \tilde{\mathfrak{g}}_{k}=\mathfrak{g}_{k} \quad \text { for } k \neq 0, \\
& \tilde{\mathfrak{g}}_{0}=\mathfrak{g}_{0}+\mathbb{C} \notin,
\end{aligned}
$$

and

$$
\tilde{\mathfrak{g}}_{+}=\sum_{k>0} \tilde{\mathfrak{g}}_{k}, \quad \tilde{\mathfrak{g}}_{-}=\sum_{k<0} \tilde{\mathfrak{g}}_{k}
$$

Then

$$
\tilde{\mathbf{g}}=\tilde{\mathbf{g}}-\oplus \tilde{\mathbf{g}}_{0} \oplus \tilde{\mathbf{g}}_{+} .
$$

Definition. Let $U(\tilde{\mathfrak{g}})$ be the universal enveloping algebra of $\tilde{\mathfrak{g}}$ and $\Lambda \in \tilde{\mathfrak{g}}_{0}{ }^{*}$, the dual space of $\tilde{\mathfrak{g}}_{0}$. Let $J(\Lambda)$ be the left ideal of $U(\tilde{\mathfrak{g}})$ generated by $\tilde{\mathfrak{g}}_{+}$and

$$
\left\{X-\Lambda(X) \mid X \in \tilde{\mathfrak{g}}_{0}\right\} \text {, }
$$

where we identify 1 with the identity of $U(\tilde{\mathfrak{g}}) . M(\Lambda):=U(\tilde{\mathfrak{g}}) / J(\Lambda)$ is called a Verma module. Any quotient of $M(\Lambda)$ is called a highest weight module of $\tilde{\mathfrak{g}}$ of highest weight $\Lambda$. 
By the Poincaré-Birkhoff-Witt theorem, $M(\Lambda)$ has basis

$$
L_{-n}^{k_{n, 1}} \ldots L_{-n}^{k_{n, s_{n}}} \ldots L_{-1}^{k_{1,1}} \ldots L_{-1}^{k_{1, s_{1}}} v_{+}
$$

where

$$
k_{j, 1} \geqq k_{j, 2} \geqq \cdots \geqq k_{j, s_{j}}, \quad 1 \leqq j \leqq n, \quad n \in \mathbb{Z}_{\geqq 0},
$$

and $v_{+}$is the image of 1 in $M(\Lambda)$.

Note that $\left[L_{0}^{1}, L_{n}^{s}\right]=n L_{n}^{s}$, for all $n \in \mathbb{Z}$ and $s \in \mathbb{Z}_{\geqq 0}$. We decompose $M(\Lambda)$ as a sum of eigenspaces of $L_{0}^{1}$ in the following.

For any $\mu \in \mathbb{C}$, define

$$
M^{\mu}:=\left\{u \in M(\Lambda) \mid L_{0}^{1} u=\mu u\right\},
$$

i.e. $M^{\mu}$ is the eigenspace of $L_{0}^{1}$ of eigenvalue $\mu$.

Since

$$
\begin{aligned}
& L_{0}^{1} \cdot L_{-n}^{k_{n, 1}} \ldots L_{-n}^{k_{n, s_{n}}} \ldots L_{-1}^{k_{1,1}} \ldots L_{-1}^{k_{1, s_{1}}} v_{+} \\
& \quad=\left(\lambda-\left(n s_{n}+(n-1) s_{(n-1)}+\cdots+s_{1}\right)\right) L_{-n}^{k_{n, 1}} \ldots L_{-n}^{k_{n, s_{n}}} \ldots L_{-1}^{k_{1,1}} \ldots L_{-1}^{k_{1, s_{1}}} v_{+},
\end{aligned}
$$

for any basis element of $M(\Lambda)$, where $\lambda=\Lambda\left(L_{0}^{1}\right)$, it is easy to see that $M(\Lambda)=$ $\sum_{k \geqq 0} M^{\lambda-k}$ and $M^{\lambda}=\mathbb{C} v_{+}$. If $N \subseteq M(\Lambda)$ is a proper $\tilde{\mathfrak{g}}$-module, then $N \cap M^{\lambda}=0$. Hence $M(\Lambda)$ is indecomposable and contains a unique maximal proper submodule $N(\Lambda)$. Let $V(\Lambda):=M(\Lambda) / N(\Lambda)$. Then $V(\Lambda)$ is the unique irreducible highest weight module of highest weight $\Lambda$. Hence we proved the following proposition.

Proposition 1. (i) $M(\Lambda)=\sum_{k \in \mathbb{Z}_{\geq 0}} M^{\lambda-k}, M^{\lambda}=\mathbb{C} v_{+}$, and $M(\Lambda)$ is indecomposable.

(ii) $M(\Lambda)$ has a unique proper maximal submodule $N(\Lambda)$ and $V(\Lambda)=$ $M(\Lambda) / N(\Lambda)$ is the unique irreducible highest weight module of highest weight $\Lambda$.

Define a total order $<$ on $\left\{L_{m}^{r} \mid m \in \mathbb{Z}, r \in \mathbb{Z}_{\geqq 0}\right\} \cup\{\phi\}$ as follows:

iff

$$
L_{m}^{r}<L_{n}^{s}
$$

$$
m<n
$$

or

$$
m=n, \quad r<s
$$

And

$$
L_{0}^{k}<\phi<L_{1}^{0} \quad \forall k \in \mathbb{Z} .
$$

Then an element of $U(\tilde{\mathfrak{g}})$ is a linear combination of elements of the form

$$
R=L_{-j_{1}}^{k_{1}} L_{-j_{2}}^{k_{2}} \ldots L_{-j_{s}}^{k_{s}}\left(L_{0}^{0}\right)^{r_{0}} \ldots\left(L_{0}^{k}\right)^{r_{n}} \phi^{l} L_{i_{1}}^{l_{1}} \ldots L_{i_{t}}^{l_{t}},
$$

where $L_{-j_{1}}^{k_{1}} \leqq \cdots \leqq L_{-j_{s}}^{k_{s}}<L_{0}^{0}, \phi<L_{i_{1}}^{l_{1}} \leqq \cdots \leqq L_{i_{t}}^{l_{t}}$. For $u \in M(\Lambda)$, define $\langle u\rangle$ to be the coefficient of the highest weight vector $v_{+}$in the expansion of $u$ with respect to $M(\Lambda)=\sum_{k \in \mathbb{Z}_{\geq 0}} M^{\lambda-k}$. If we extend the anti-involution $\omega$ of $\tilde{\mathfrak{g}}$ to $U(\tilde{\mathfrak{g}})$ by $\omega(X Y)=\omega(Y) \omega(X)$, then we have $\left\langle\omega(R) v_{+}\right\rangle=\overline{\left\langle R v_{+}\right\rangle}$provided that $\Lambda\left(L_{0}^{k}\right) \in \mathbb{R}$ for $k=0,1,2 \ldots$ and $\Lambda(\phi) \in \mathbb{R}$.

\section{Proposition 2.}

(i) Assume that $\Lambda\left(L_{0}^{k}\right) \in \mathbb{R}$ for all $k \in \mathbb{Z} \geqq 0$ and $\Lambda(\phi) \in \mathbb{R}$. Then $M(\Lambda)$ carries a unique contravariant hermitian form $\langle\cdot, \cdot\rangle$ such that $\left\langle v_{+}, v_{+}\right\rangle=1$;

(ii) $\left\langle M^{\lambda-k}, M^{\lambda-l}\right\rangle=0$ if $k \neq l$; 
(iii) $\operatorname{ker}\langle\cdot, \cdot\rangle=N(\Lambda)$. Hence $V(\Lambda)$ carries a unique contravariant hermitian form such that $\langle v, v\rangle=1$ and this form is non-degenerate, where $v=v_{+}+N(\Lambda)$.

Proof. (i), (ii). For any monomials $P\left(v_{+}\right)=L_{-j_{1}}^{k_{1}} \ldots L_{-j_{s}}^{k_{s}} v_{+}$and $Q\left(v_{+}\right)=$ $L_{-i_{1}}^{l_{1}} \ldots L_{-i_{t}}^{l_{l} v_{+}}$, define $\left\langle P\left(v_{+}\right), Q\left(v_{+}\right)\right\rangle:=\left\langle\omega(P) Q\left(v_{+}\right)\right\rangle$. This is a well defined contravariant hermitian form on $M(\Lambda)$ (see $[\mathrm{S}, \mathrm{KR}$ or MoPi]). Moreover, $\left\langle P\left(v_{+}\right), Q\left(v_{+}\right)\right\rangle=0$, if $j_{1}+j_{2}+\cdots+j_{s} \neq i_{1}+i_{2}+\cdots+i_{t}$.

(iii). By definition,

$$
\operatorname{ker}\langle\cdot, \cdot\rangle=\{u \in M(\Lambda) \mid\langle u, w\rangle=0 \quad \forall w \in M(\Lambda)\} .
$$

Clearly $k e r\langle\cdot, \cdot\rangle$ is a proper submodule of $M(\lambda)$. Moreover, if $V \subseteq M(\Lambda)$ is a submodule and $P\left(v_{+}\right) \in V, Q\left(v_{+}\right) \in M(\Lambda)$, then $\omega(Q) P\left(v_{+}\right) \in V$. So if $V$ is a proper submodule of $M(\Lambda)$, then $\left\langle\omega(Q) P\left(v_{+}\right)\right\rangle=0$, i.e. $\left\langle P\left(v_{+}\right), Q\left(v_{+}\right)\right\rangle=0$. Hence $P\left(v_{+}\right) \in \operatorname{ker}\langle\cdot, \cdot\rangle$ and $V \subseteq \operatorname{ker}\langle\cdot, \cdot\rangle$. So we proved that

$$
\operatorname{ker}\langle\cdot, \cdot\rangle=N(\Lambda) \text {. }
$$

An important question is when $\langle\cdot, \cdot\rangle$ is non-negative on $M(\Lambda)$, hence positive definite on $V(\Lambda)$. For Virasoro algebra $\tilde{\mathfrak{g}}\{x\}$, D. Friedan, Z. Qiu, and S. Shenker [FQS], and R. Langlands [L] gave a necessary condition for the corresponding form $\langle\cdot, \cdot\rangle$ on Verma module to be non-negative. P. Goddard, A. Kent and D. Olive [GKO] proved that the condition is also sufficient. For $\tilde{\mathfrak{g}}$, even though we still do not know examples for which $\langle\cdot, \cdot\rangle$ are non-negative on $M(\Lambda)$, we can prove the following necessary condition. First, for $c \in \mathbb{R}, \underline{h}=\left(h_{0}, h_{1}, h_{2}, \ldots\right) \in \mathbb{R}^{\infty}$, we define an infinite real matrix $A(\underline{h}, c)$ as follows:

$$
A(\underline{h}, c)=\left(A_{i j}(\underline{h}, c)\right)_{i, j=1,2, \ldots},
$$

where

$$
A_{i j}(\underline{h}, c):=\sum_{l=0}^{i+j}\left(\begin{array}{c}
i+j \\
l
\end{array}\right)\left(\frac{1}{2}\right)^{l}\left((-1)^{l}-1\right) h_{i+j-l}-\left(\frac{1}{2}\right)^{i+j} c
$$

Clearly,

$$
A_{i j}(\underline{h}, c)=A_{j i}(\underline{h}, c)
$$

Proposition 3. Let $\Lambda \in \tilde{\mathfrak{g}}_{0}^{*}$ be such that $h_{i}=\Lambda\left(L_{0}^{i}\right) \in \mathbb{R} \forall i \in \mathbb{Z}_{\geqq 0}$ and $c=\Lambda(\phi) \in \mathbb{R}$. Then a necessary condition for $\langle\cdot, \cdot\rangle$ to be non-negative on $M(\Lambda)$ is that $A(\underline{h}, c)$ is positive semidefinite.

Proof. For $\left(a_{0}, a_{1}, \ldots, a_{r}\right) \in \mathbb{R}^{r+1}$, if $\langle\cdot, \cdot\rangle$ is non-negative, then

$$
\left\langle\left(\sum_{i=1}^{r} a_{i} L_{-1}^{i}\right) v_{+},\left(\sum_{i=1}^{r} a_{i} L_{-1}^{i}\right) v_{+}\right\rangle \geqq 0,
$$

i.e.

$$
\left\langle v_{+}, \sum_{i=1}^{r} \sum_{j=1}^{r} a_{i} a_{j}\left[L_{1}^{i}, L_{-1}^{j}\right] v_{+}\right\rangle \geqq 0
$$


Since

$$
\begin{gathered}
{\left[L_{1}^{i}, L_{-1}^{j}\right]=\sum_{l=0}^{i+j}\left(\begin{array}{c}
i+j \\
l
\end{array}\right)\left(\frac{1}{2}\right)^{l}\left((-1)^{l}-1\right) L_{0}^{i+j-l}-\left(\frac{1}{2}\right)^{i+j} \phi,} \\
\sum_{i} \sum_{j} a_{i} a_{j}\left(\sum_{l=0}^{i+j}\left(\begin{array}{c}
i+j \\
l
\end{array}\right)\left(\frac{1}{2}\right)^{l}\left((-1)^{l}-1\right) h_{i+j-l}-\left(\frac{1}{2}\right)^{i+j} c\right) \geqq 0 .
\end{gathered}
$$

This is

$$
\sum_{i} \sum_{j} a_{i} a_{j} A_{i j}(\underline{h}, c) \geqq 0
$$

or

$$
\left(\begin{array}{llllll}
a_{0} & a_{1} & \ldots & a_{r} & 0 & \ldots
\end{array}\right) A(\underline{h}, c)\left(\begin{array}{c}
a_{0} \\
a_{1} \\
\vdots \\
a_{r} \\
0 \\
\vdots
\end{array}\right) \geqq 0 .
$$

So $A(\underline{h}, c)$ is positive semidefinite.

Finally, we consider the highest weight modules of $\tilde{\mathfrak{g}}\{0,1\}$. Take

$$
\left\{L_{m}^{1}, L_{m}^{0} \mid \forall m \in \mathbb{Z}\right\} \cup\{\notin\}
$$

as a basis. Then

$$
\tilde{\mathfrak{g}}\{0,1\}=\sum_{m \in \mathbb{Z}} \mathbb{C} L_{m}^{1}+\sum_{m \in \mathbb{Z}} \mathbb{C} L_{m}^{0}+\mathbb{C} \phi,
$$

and

$$
\begin{aligned}
& {\left[L_{m}^{1}, L_{n}^{1}\right]=(n-m) L_{m+n}^{1}-\frac{1}{12}\left(m^{3}+2 m\right) \delta_{m+n, 0} \phi,} \\
& {\left[L_{m}^{1}, L_{n}^{0}\right]=n L_{m+n}^{0}-\frac{m}{2} \delta_{m+n, 0} \phi,} \\
& {\left[L_{m}^{0}, L_{n}^{0}\right]=-m \delta_{m+n, 0} \phi .}
\end{aligned}
$$

Let

Then

$$
\begin{aligned}
L_{m} & =-L_{m}^{1}-\frac{1}{8} \delta_{m, 0} \phi, \\
A_{m} & =-L_{m}^{0}-\frac{1}{2} \delta_{m, 0} \phi, \\
\phi_{1} & =-\phi .
\end{aligned}
$$

$$
\begin{aligned}
& {\left[L_{m}, L_{n}\right]=(n-m) L_{m+n}+\frac{m^{3}-m}{12} \delta_{m+n, 0} \phi_{1},} \\
& {\left[L_{m}, A_{n}\right]=-n A_{m+n},} \\
& {\left[A_{m}, A_{n}\right]=m \delta_{m+n, 0} \phi_{1},}
\end{aligned}
$$


and

$$
\omega\left(L_{m}\right)=L_{-m}, \quad \omega\left(A_{m}\right)=A_{-m}, \quad \omega\left(\phi_{1}\right)=\phi_{1} .
$$

Let

$$
\begin{aligned}
& \tilde{\mathfrak{g}}\{0,1\}_{+}=\sum_{m>0} \mathbb{C} L_{m}+\sum_{m>0} \mathbb{C} A_{m}, \\
& \tilde{\mathfrak{g}}\{0,1\}_{-}=\sum_{m<0} \mathbb{C} L_{m}+\sum_{m<0} \mathbb{C} A_{m},
\end{aligned}
$$

and

$$
\tilde{\mathfrak{g}}\{0,1\}_{0}=\mathbb{C} L_{0}+\mathbb{C} A_{0}+\mathbb{C} \phi_{1} .
$$

Then

$$
\tilde{\mathfrak{g}}\{0,1\}=\tilde{\mathfrak{g}}\{0,1\}_{+} \oplus \tilde{\mathfrak{g}}\{0,1\}_{0} \oplus \tilde{\mathfrak{g}}\{0,1\} \ldots
$$

For $\Lambda \in \mathfrak{g}\{0,1\}_{0}{ }^{*}$, as in the case of $\tilde{\mathfrak{g}}$, we have Verma module $M(\Lambda)$, the unique maximal proper submodule $N(\Lambda)$ and $V(\Lambda):=M(\Lambda) / N(\Lambda)$. Moreover, if

$$
h:=\Lambda\left(L_{0}\right), \quad a:=\Lambda\left(A_{0}\right), \quad c:=\Lambda\left(\phi_{1}\right) \in \mathbb{R},
$$

there exists a contravariant hermitian form $\langle\cdot, \cdot\rangle$ on $M(\Lambda)$ such that $\operatorname{ker}\langle\cdot, \cdot\rangle=N(\Lambda)$.

Proposition 4. Let

$$
\begin{gathered}
\tilde{\mathfrak{g}}\{0,1\}=\sum_{m \in \mathbb{Z}} \mathbb{C} L_{m}+\sum_{m \in \mathbb{Z}} \mathbb{C} A_{m}+\mathbb{C} \phi_{1} \\
\Lambda \in\left(\mathbb{C} L_{0}+\mathbb{C} A_{0}+\mathbb{C} \phi_{1}\right)^{*}
\end{gathered}
$$

be such that

$$
h=\Lambda\left(L_{0}\right), \quad a=\Lambda\left(A_{0}\right), \quad c=\Lambda\left(\phi_{1}\right) \in \mathbb{R} .
$$

Then a necessary condition for $\langle\cdot, \cdot\rangle$ to be non-negative on $M(\Lambda)$ is

$$
(h, c) \geqq(0,1) \text {, }
$$

or

$$
(h, c)=\left(h_{m}^{r, s}, c_{m}\right) \quad m \geqq 0
$$

where

$$
\begin{aligned}
h_{m}^{r, s} & =\frac{((m+3) r-(m+2) s)^{2}-1}{4(m+2)(m+3)} \\
c_{m} & =1-\frac{6}{(m+2)(m+3)} \quad 1 \leqq s \leqq r \leqq m+1 .
\end{aligned}
$$

And for any given $(h, c)$ satisfying $(i)$ or $(i i)$,

$$
-\sqrt{2 h c} \leqq a \leqq \sqrt{2 h c} .
$$

Proof. The conditions (i) and (ii) on $(h, c)$ are given by [FQS] and [L]. For any given pair $(h, c)$ satisfying (i) and (ii), since $\forall \alpha \in \mathbb{R}$,

$$
\left\langle\left(L_{-1}+\alpha A_{-1}\right) v_{+} \mid\left(L_{-1}+\alpha A_{-1}\right) v_{+}\right\rangle \geqq 0,
$$


we have

$$
2 h+2 \alpha a+\alpha^{2} c \geqq 0,
$$

or

$$
2 \alpha a \geqq-2 h-\alpha^{2} c
$$

Thus

$$
\begin{cases}a \geqq \frac{-h}{\alpha}-\frac{\alpha c}{2} \quad \forall \alpha \in \mathbb{R}_{>0} \\ a \leqq-\frac{h}{\alpha}-\frac{\alpha c}{2} \quad \forall \alpha \in \mathbb{R}_{<0} .\end{cases}
$$

Define $f(\alpha):=-\frac{h}{\alpha}-\frac{\alpha c}{2}$ and let

$$
f^{\prime}(\alpha)=\frac{h}{\alpha^{2}}-\frac{c}{2}=0
$$

We get $\alpha= \pm \sqrt{\frac{2 h}{c}}$ and

$$
-\sqrt{2 h c}=f\left(\sqrt{\frac{2 h}{c}}\right) \leqq a \leqq f\left(-\sqrt{\frac{2 h}{c}}\right)=\sqrt{2 h c} .
$$

Acknowledgement. This work constitutes part of my Ph.D. thesis in the University of Alberta. I would like to express my sincere gratitute to my supervisor R.V. Moody for his guidance and support.

\section{References}

[AK] Aoyama, S., Kodama, Y.: A generalized Sato equation and the $W_{\infty}$ algebra, Phys. Letters B 278, 56-62 (1992)

[BM] Benkart, G.M., Moody, R.V.: Derivations, central extentions and affine Lie algebras. Algebras, Groups and Geometries 3 (1986)

[C] Chen, L.: Differential operator Lie algebras on the ring of Laurent polynomials. Ph.D Thesis, University of Alberta, Canada (1993)

[CP] Chari, V., Pressley, A.: Unitary representations of the Virasoro algebra and a conjecture of Kao. Composito Math. 67, 315-342 (1988)

[FQS] Frieden, D., Qiu, Z., Shenker, S.: Details of the non-unitarity proof for highest weight representations of the Virasoro algebra. Commun. Math. Phys. 107, 535-542 (1986)

[G] Garland, H.: The arithmetic theory of loop groups. Publ. Math. IHES 52, 5-136 (1980)

[GKO] Goddard, P., Kent, A., Olive, D.: Unitary representations of the Virasoro and superVirasoro algebras. Commun. Math. Phys. 103, 105-119 (1986).

[K1] Kac, V.: Infinite dimentinal Lie algebras. 3rd edition, Cambridge: Cambridge University Press, 1990

[K2] Kac, V.: Some problem on infinite dimensional Lie algebras and their representations. Lecture Note in Math. 933 (1982)

[Kap] Kaplansky, I.: The Virasoro algebra. Commun. Math. Phys. 86 (1982)

[KR] Kac, V., Raina, A.: Highest weight representations of infinite dimentional Lie algebra. Singapore: World Scientific, 1989

[KS] Kaplansky, I., Santharoubane, L.J.: Harrish-Chandra modules over the Virasoro algebra. MSRI Publications Vol. 5, Berlin, Heidelberg, New York: Springer 1985, pp. $217-231$ 
[L] Langlands, R.: On unitary representations of the Virasoro algebra.

[Li] Li, Wang-Lai: 2-cocycles on the algebra of differential operators, J. Algebra 122, 64-80 (1989)

[Ma] Mathieu, O.: Classification of Harish-Chandra modules over the Virasoro Lie algebra. Invent. Math. 107, 225-234 (1992)

[MP] Martin, C., Piard, A.: Indecomposable modules over the Virasoro Lie algebra and a conjecture of V. Kac. Commun. Math. Phys. 137, 109-132 (1991)

[MoPi] Moody, R., Pianzola, A.: Lie algebras with triangular decompositions, (to appear)

[PRS1] Pope, C.N., Romans, L.J., Shen, X.: The complete structure of $W_{\infty}$. Phys. Lett. B 236, No. 2 (1990)

[PRS2] Pope, C.N., Romans, L.J., Shen, X.: A new higher spin algebra and the longstar product. Phys. Lett. B 242, No. 3, 4 (1990)

[PRS3] Pope, C.N., Romans, L.J., Shen, X.: $W_{\infty}$ and the Racahwigner algebra, (to appear)

[PRS4] Pope, C.N., Romans, L.J., Shen, X.: Ideals of Kac-Moody algebras and realizations of $W_{\infty}$. Phys. Lett. B 245, No. 1 (1990)

[PS] Pope, C.N., Shen, X.: Higher-spin theories, $W_{\infty}$ algebras and their superextentions. Phys. Lett. B 236, No. 1 (1990)

[Sa] Sato, Sato: Lecture Notes in Num. Appl. Anal., Vol. 5, 1982, pp. 259-271

[Sh] Shapavolov, N.N.: On a bilinear form on the universal enveloping algebra of a complex semisimple Lie algebra, Funct. Anal. Appl. (English translation) 6, 307-312 (1972)

[SW] Shen, X., Wang, X.J.: Bosonisation of the complex-boson realizations of $W_{\infty}$. Phys. Lett. B 278, 63-71 (1992)

Communicated by M. Jimbo 
